\title{
Measuring evidence: a probabilistic approach to an extension of Belnap-Dunn logic
}

Article in Synthese - March 2020

DOI: 10.1007/s11229-020-02571-w

\section{CITATIONS}

3

3 authors:

Abilio Rodrigues

Federal University of Minas Gerais

22 PUBLICATIONS 64 CITATIONS

SEE PROFILE

Walter Carnielli

Centre for Logic, Epistemology and the History of Science

70 PUBLICATIONS 165 CITATIONS

SEE PROFILE

Some of the authors of this publication are also working on these related projects:

Project Brouwerian Relevance Logic View project

Project Paraconsistent probabilities and possibilistic measures View project
READS

33

Juliana Bueno-Soler

Universidade Federal do $A B C$ (UFABC)

16 PUBLICATIONS 72 CITATIONS

SEE PROFILE 


\title{
Measuring evidence: a probabilistic approach to an extension of Belnap-Dunn Logic *
}

\author{
Abilio Rodrigues ${ }^{1}$, Juliana Bueno-Soler ${ }^{2}$, Walter Carnielli² \\ ${ }^{1}$ Federal University of Minas Gerais \\ abilio.rodrigues@gmail.com \\ ${ }^{2}$ University of Campinas \\ juliana@ft.unicamp.br \\ walter.carnielli@gmail.com
}

February 27, 2020

\begin{abstract}
This paper introduces the logic of evidence and truth $L E T_{F}$ as an extension of the Belnap-Dunn four-valued logic FDE. $L E T_{F}$ is a slightly modified version of the logic $L E T_{J}$, presented in Carnielli and Rodrigues (2017). While $L E T_{J}$ is equipped only with a classicality operator o, $L E T_{F}$ is equipped with a non-classicality operator $\bullet$ as well, dual to $\circ$. Both $L E T_{F}$ and $L E T_{J}$ are logics of formal inconsistency and undeterminedness in which the operator o recovers classical logic for propositions in its scope. Evidence is a notion weaker than truth in the sense that there may be evidence for a proposition $\alpha$ even if $\alpha$ is not true. As well as $L E T_{J}$, $L E T_{F}$ is able to express preservation of evidence and preservation of truth. The primary aim of this paper is to propose a probabilistic semantics for $L E T_{F}$ where statements $P(\alpha)$ and $P(\circ \alpha)$ express, respectively, the amount of evidence available for $\alpha$ and the degree to which the evidence for $\alpha$ is expected to behave classically - or non-classically for $P(\bullet \alpha)$. A probabilistic scenario is paracomplete when $P(\alpha)+P(\neg \alpha)<1$, and paraconsistent when $P(\alpha)+P(\neg \alpha)>1$, and in both cases, $P(\circ \alpha)<1$. If $P(\circ \alpha)=1$, or $P(\bullet \alpha)=0$, classical probability is recovered for $\alpha$. The proposition $\circ \alpha \vee \bullet \alpha$, a theorem of $L E T_{F}$, partitions what we call the information space, and thus allows us to obtain some new versions of known results of standard probability theory.
\end{abstract}

${ }^{*}$ The authors acknowledge support from the National Council for Scientific and Technological Development (CNPq, Brazil) under research grants 311911/2018-8, 308077/2018-0, and 307376/2018-4. The first author has also been supported by the CNPq fellowship grant 204479/2017-71 for a research stay at the University of Oxford, 2018. The authors owe a great debt to the referees for the careful reading of an earlier version of this text, which lead to relevant suggestions for improving it. 


\section{Introduction}

In Carnielli and Rodrigues (2017) two paraconsistent and paracomplete formal systems were presented, the Basic Logic of Evidence $(B L E)$ and the Logic of Evidence and Truth $\left(L E T_{J}\right)$. BLE ends up being equivalent to Nelson's wellknown logic $N_{4}$ but has been conceived to express preservation of evidence instead of truth. Scenarios with conflicting evidence - that is, non-conclusive evidence for the truth and the falsity of $\alpha$ - as well as scenarios with no evidence at all about $\alpha$ are possible, so neither explosion nor excluded middle hold in $B L E$. $L E T_{J}$ is an extension of $B L E$ equipped with a classicality operator $\circ$. When $\circ \alpha$ holds, classical negation - and so full classical logic - for $\alpha$ is recovered. According to the intended interpretation, $\circ \alpha$ in $L E T_{J}$ means that there is conclusive evidence for the truth or falsity of $\alpha$, so the truth-value of $\alpha$ has been established as true or false.

Let us call $\vdash_{C}$ and $\vdash_{B L E}$, respectively, the relation of logical consequence in classical logic and in BLE. Classical consequence is defined in terms of preservation of truth: $\Gamma \vdash_{C} \alpha$ just in case there is no model $M$ such that all propositions of $\Gamma$ are true in $M$, but $\alpha$ is not true in $M$. The intended interpretation of $B L E$, on the other hand, is not based on preservation of truth, but rather on preservation of evidence: $\Gamma \vdash_{B L E} \alpha$ means that the availability of evidence for the premises in $\Gamma$ implies that there is also evidence available for $\alpha$. Classical logic and $B L E$, therefore, express different properties of propositions: truth and availability of evidence. The logic $L E T_{J}$, in its turn, is able to express preservation of evidence and preservation of truth - it 'combines', in one and the same formal system, the relations $\vdash_{C}$ and $\vdash_{B L E}$. The operator $\circ$ works like a context switch that divides propositions into those that have a classical and those that have a non-classical behavior, and $B L E$ is the underlying logic of the latter.

Adequate valuation semantics and decision procedures for $B L E$ and $L E T_{J}$ have been proposed. These semantics, however, are only able to express the fact that a given proposition $\alpha$ has or does not have evidence available by attributing, respectively, the semantic value 1 or 0 to $\alpha$. Evidence, thus, is treated from a purely qualitative point of view. A question that presents itself is whether the amount of evidence available for a given proposition $\alpha$ could be quantified. Here we give a positive answer to this question.

The aim of this paper is to propose a probabilistic semantics for a modified version of $L E T_{J}$ obtained by dropping the implication symbol $\rightarrow$ and adding a non-classicality operator $\bullet$ dual to $\circ$. While $\circ \alpha$ implies that $\alpha$ behaves classicaly, a non-classical behavior of $\alpha$ implies $\bullet \alpha$. The logic so obtained is an extension of the well-known logic of First-Degree Entailment $(F D E)$, and we call it $L E T_{F}$, the Logic of Evidence and Truth based on FDE. As well as $L E T_{J}, L E T_{F}$ is suitable to an intuitive reading in terms of evidence and truth.

In order to capture this idea of preservation of degrees of evidence a nonclassical notion of probability will be employed. The probabilistic semantics proposed here follows the ideas presented in Bueno-Soler and Carnielli (2016. 2017). Let $P(\alpha)=\epsilon$ mean that $\epsilon$ is the measure of evidence available for $\alpha$. We call a probabilistic scenario paracomplete when $P(\alpha)+P(\neg \alpha)<1$, and 
paraconsistent when $P(\alpha)+P(\neg \alpha)>1$. These two cases can be explained, respectively, as 'too little information' and 'too much information' about $\alpha{ }^{1}$ In both cases, $P(\circ \alpha)<1$, which means that the probability measures of $\alpha$ and $\neg \alpha$ are not behaving classically. So, $P(\circ \alpha)<1$ means that the information available about $\alpha$ is not reliable, and something must be wrong. If $P(\circ \alpha)=1$, standard probability is recovered for $\alpha$.

With the purpose of understanding the probabilistic semantics proposed here better, we adopt a notion of information space instead of the standard notion of sample space. The intuitive idea is to collect all the relevant information about a proposition $\alpha$ (or about a set of propositions $\Gamma$ ) and the corresponding measures of evidence. So, roughly speaking, an information space is constituted by propositions that represent evidence that can be non-conclusive, contradictory or incomplete, more reliable or less reliable, and sometimes conclusive (we return to this point in Section 4.3 below). Such a notion of information space requires a generalization of the notion of a partition, and consequently allows us to obtain generalized versions of standard results of probability theory such as total probability theorem and Bayes' rule $2^{2}$

The remainder of this paper is organized in four sections. Section 2 is dedicated to the logic FDE. It is shown that FDE is suited to an interpretation in terms of preservation of evidence. We also present adequate valuation semantics and a decision procedure for $F D E$. In Section 3, $F D E$ is extended to $L E T_{F}$, and an adequate semantics, a decision procedure, and some relevant results are presented and discussed. In Section 4 , a probabilistic semantics for $L E T_{F}$ is defined, and paraconsistent and paracomplete versions of total probability theorems and Bayes' rule are also presented and discussed. Finally, in Section 5, we discuss some points related to the topics of this paper that could be developed further.

\section{$2 F D E$ as a logic of preservation of evidence}

The inference rules of $B L E$ were obtained by asking whether an inference rule preserves evidence. Since evidence can be incomplete (no evidence at all) and contradictory (conflicting evidence), explosion and excluded middle do not hold. In $B L E$, when $\alpha$ (resp. $\neg \alpha$ ) holds, the intended meaning is that there is evidence for the truth (resp. falsity) of $\alpha$. Evidence that $\alpha$ is true and evidence that $\alpha$

\footnotetext{
${ }^{1}$ The connections between the notions of evidence and information will be explained in Section 2.2 .1

${ }^{2}$ Our approach differs from the so-called Dempster-Shafer (DS) theory of evidence, developed by Glenn Shafer in Shafer (1976) and based on earlier work of Arthur Dempster. DS is focused on degrees of belief and degrees of plausibility. As Lofti Zadeh points out in his review (Zadeh, 1984), the DS theory falls short as a useful tool for the management of uncertainty (even for expert systems, for which it was designed). Our approach, as we try to make clear throughout this paper, uses probabilistic semantics intended to quantify the evidence attributed to a proposition and introduces a new logic with an intuitive reading in terms of preservation of evidence and truth. That is the reason we cannot rely on the DS 'mathematical' theory of evidence: it is not so attractive as it seems to be at first glance, and lacks the features we are interested in.
} 
is false are independent of each other, and are treated as such by the formal system. $B L E$ can express the following four scenarios:

1. Only evidence that $\alpha$ is true: $\alpha$ holds, $\neg \alpha$ does not hold.

2. Only evidence that $\alpha$ is false: $\neg \alpha$ holds, $\alpha$ does not hold.

3. No evidence at all: neither $\alpha$ nor $\neg \alpha$ hold.

4. Conflicting evidence: both $\alpha$ and $\neg \alpha$ hold ${ }^{3}$

Evidence for a proposition $\alpha$ is explained in Carnielli and Rodrigues (2017, Section 2) as reasons for believing in $\alpha$, but these reasons may be non-conclusive or even wrong, and do not imply the truth of $\alpha$, nor the belief in $\alpha$. Thus, evidence is a notion weaker than truth in the sense that there may be evidence for a proposition $\alpha$ even if $\alpha$ is not true. Below, in Section 2.2.1, starting from the notion of information proposed by Dunn (2008), we explain evidence in terms of a (perhaps) non-conclusive justification added to a proposition $\alpha$ or, as Fitting (2016b) puts it, "justifications that might be wrong" 4 Notice that the notion of evidence encompasses non-conclusive as well as conclusive evidence, and the latter is evidence that establishes the truth-value of a proposition $\alpha$.

The logic of First-Degree Entailment $(F D E)$ is a paraconsistent and paracomplete propositional logic in a language with conjunction, disjunction, and negation, with no theorems nor bottom particles (cf. Anderson and Belnap, 1963 , 1975, Anderson et al., 1992, Belnap, 1977a b; Dunn, 1976). FDE is a fragment of $B L E / N 4$, obtained by dropping the implication symbol and the corresponding rules, and it can be interpreted in terms of preservation of evidence, as well as $B L E$ - the four scenarios above clearly correspond to the four truth-values proposed by Belnap (1977a b) (we return to this point in Section 2.2 below) $!^{5}$

Definition 1. The Logic of First-Degree Entailment (FDE)

Let $L_{1}$ be a language with a denumerable set of sentential letters $\left\{p_{1}, p_{2}, p_{3}, \ldots\right\}$, the set of connectives $\{\neg, \wedge, \vee\}$, and parentheses. The set of formulas of $L_{1}$

\footnotetext{
${ }^{3}$ The expression ' $\alpha$ holds/does not hold' here means that $\alpha$ holds/does not hold in $B L E$. So, here, it does not mean that $\alpha$ is true/false.

4 Fitting (2016a) presents an embedding of $B L E$ into the modal logic $K X_{4}$, and an embedding of the later into the justification logic $J X_{4}$. The latter is equipped with justification terms that stand for "justification, or evidence, which may be non-factual, uncertain, or contradictory" (Fitting, 2016a p. 1159). In $J X_{4}$, ' $t: \alpha$ ' means that $\alpha$ is justified by reason $t$. The notion of evidence expressed by $K_{4} 4$ (implicit evidence) and $J_{4} 4$ (explicit evidence) is a "formal alternative" of the "informal" notion of evidence expressed by $B L E$.

${ }^{5}$ The move from $B L E$ and $L E T_{J}$ to (respectively) $F D E$ and $L E T_{F}$ has been motivated by some difficulties in interpreting the implication of $B L E$ in probabilistic terms. The implication of $B L E$ is located somewhere in between classical and intuitionistic implication: it is not classical because Peirce's Law does not hold, and it is not intuitionistic because the equivalence between $\neg(\alpha \rightarrow \beta)$ and $\alpha \wedge \neg \beta$ holds. It is not clear what would be the intuitive meaning of the attribution of a probabilistic measure to a formula $\alpha \rightarrow \beta$ of $B L E$, and how this measure would relate to the probabilistic values of $\alpha$ and $\beta$. So we decided, at least in this paper, to work with $F D E$, the implication-free fragment of $B L E$.
} 
is obtained recursively in the usual way. The logic FDE is defined over the language $L_{1}$ by the following natural deduction rules:

$$
\frac{\alpha \beta}{\alpha \wedge \beta} \wedge I \quad \frac{\alpha \wedge \beta}{\alpha} \wedge E \frac{\alpha \wedge \beta}{\beta}
$$

(1)

(1)

(1)

(1)

A

there the definition of a derivation is the usual one for natural deduction systems (see e.g. van Dalen (2008, pp. 35-36)).

Other deductive systems have already been presented for FDE (see Omori and Wansing, 2017, Section 2.2), but the natural deduction system proposed here makes the symmetry between positive and negative rules explicit: $\wedge I$ and $\neg \vee I$ are symmetrical, $\vee E$ and $\neg \wedge E$ are symmetrical, and so on. This mirrors the fact that positive and negative evidence are primitive and non-complementary notions, but have symmetric deductive behavior: the rule $\wedge I$ expresses the idea that when there is positive evidence available for both $\alpha$ and $\beta$, there is positive evidence for $\alpha \wedge \beta$, while the rule $\neg \vee I$ means that when there is negative evidence available for both $\alpha$ and $\beta$, there is negative evidence for $\alpha \vee \beta$.

\section{Theorem 2.}

Reflexivity, monotonicity, transitivity, and compactness hold for FDE.

Proof. These well-known properties of FDE can be easily proved by means of the natural deduction system above.

\subsection{Valuation semantics for $F D E$}

We now propose a non-deterministic valuation semantic for $F D E$.

Definition 3. Valuation semantics for FDE

$A$ valuation semantics for FDE is a collection of FDE-valuations defined as follows: A function $v: L_{1} \rightarrow\{0,1\}$ is a FDE-valuation if it satisfies the following clauses: 
v1. $v(\alpha \wedge \beta)=1$ iff $v(\alpha)=1$ and $v(\beta)=1$,

v2. $v(\alpha \vee \beta)=1$ iff $v(\alpha)=1$ or $v(\beta)=1$,

v3. $v(\neg(\alpha \wedge \beta))=1$ iff $v(\neg \alpha)=1$ or $v(\neg \beta)=1$,

v4. $v(\neg(\alpha \vee \beta))=1$ iff $v(\neg \alpha)=1$ and $v(\neg \beta)=1$,

v5. $v(\alpha)=1$ iff $v(\neg \neg \alpha)=1$.

Definition 4. We say that a formula $\alpha$ is a semantical consequence of $\Gamma$, $\Gamma \vDash_{F D E} \alpha$, iff for every valuation $v$, if $v(\beta)=1$ for all $\beta \in \Gamma$, then $v(\alpha)=1$.

This semantics is sound and complete, and provides a decision procedure for $F D E$. From now on, in this section, when there is no risk of ambiguity, we will just write $\vdash$ and $\vDash$ in the place of $\vdash_{F D E}$ and $\vDash_{F D E}$.

\section{Theorem 5. Soundness}

Let $\Gamma$ be a set of formulas, and $\alpha$ a formula of FDE. So, $\Gamma \vdash \alpha$ implies $\Gamma \vDash \alpha$.

Proof. The proof is routine. It shows that assuming there are sound derivations for the premise(s), the derivation obtained by the application of a rule is sound.

\section{Theorem 6. Completeness}

Let $\Gamma$ be a set of formulas, and $\alpha$ a formula of $F D E$. Then $\Gamma \vDash \alpha$ implies $\Gamma \vdash \alpha$.

Proof. Completeness can be proved by a Henkin-style proof. Given $\Gamma$ and $\alpha$ such that $\Gamma \Vdash \alpha$, a set $\Delta$ maximal w.r.t $\alpha$ can be obtained in the usual way. So, the proof of the following propositions is straightforward:

$v 1^{\prime} . \alpha \wedge \beta \in \Delta$ iff $\alpha \in \Delta$ and $\beta \in \Delta$

$v 2^{\prime} . \alpha \vee \beta \in \Delta$ iff $\alpha \in \Delta$ or $\beta \in \Delta$;

v3'. $\neg(\alpha \wedge \beta) \in \Delta$ iff $\neg \alpha \in \Delta$ or $\neg \beta \in \Delta$;

$v_{4}{ }^{\prime} . \neg(\alpha \vee \beta) \in \Delta$ iff $\neg \alpha \in \Delta$ and $\neg \beta \in \Delta$;

$v 5^{\prime} . \alpha \in \Delta$ iff $\neg \neg \alpha \in \Delta$.

Let $v$ be the mapping from the language $L_{1}$ to $\{0,1\}$ defined as follows: for every $\gamma \in L_{1}, v(\gamma)=1$ iff $\gamma \in \Delta$. $v$ is a valuation for $F D E$ such that: for every $\beta \in \Gamma, v(\beta)=1$, since $\Gamma \subseteq \Delta$; but $v(\alpha)=0$, since $\alpha \notin \Delta(\Delta$ is maximal w.r.t. $\alpha)$. Therefore, $\Gamma \notin \alpha$.

The valuation semantics proposed in Definition 3 is non-deterministic in the sense that the semantic value of a formula $\neg \alpha$ is not a function of the value of 
$\alpha$. The possible values a formula can receive are given by quasi-matrices $\sqrt[6]{ }$ In Example 7 below, we illustrate how a quasi-matrix works.

Example 7. In FDE:

$$
\begin{aligned}
& \text { 1. } p, \neg p \vee q \sharp q ; \\
& \text { 2. } p, \neg(p \wedge q) \vDash \neg q ; \\
& \text { 3. } \neg p \wedge \neg q \Rightarrow \vDash \neg(p \vee q) \text {; } \\
& \text { 4. } \neg p \vee \neg q \Rightarrow \vDash \neg(p \wedge q) \text {. }
\end{aligned}
$$

\begin{tabular}{|c|c|c|c|c|c|c|c|c|c|c|c|c|c|c|c|c|}
\hline$p$ & \multicolumn{8}{|c|}{0} & \multicolumn{8}{|c|}{1} \\
\hline$\neg p$ & \multicolumn{4}{|c|}{0} & \multicolumn{4}{|c|}{1} & \multicolumn{4}{|c|}{0} & \multicolumn{4}{|c|}{1} \\
\hline$q$ & \multicolumn{2}{|c|}{0} & \multicolumn{2}{|c|}{1} & \multicolumn{2}{|c|}{0} & \multicolumn{2}{|c|}{1} & \multicolumn{2}{|c|}{0} & \multicolumn{2}{|c|}{1} & \multicolumn{2}{|c|}{0} & \multicolumn{2}{|c|}{1} \\
\hline$\neg q$ & 0 & 1 & 0 & 1 & 0 & 1 & 0 & 1 & 0 & 1 & 0 & 1 & 0 & 1 & 0 & 1 \\
\hline$\neg p \vee q$ & 0 & 0 & 1 & 1 & 1 & 1 & 1 & 1 & 0 & 0 & 1 & 1 & 1 & 1 & 1 & 1 \\
\hline$\neg(p \wedge q)$ & 0 & 1 & 0 & 1 & 1 & 1 & 1 & 1 & 0 & 1 & 0 & 1 & 1 & 1 & 1 & 1 \\
\hline valuation & 1 & 2 & 3 & 4 & 5 & 6 & 7 & 8 & 9 & 10 & 11 & 12 & 13 & 14 & 15 & 16 \\
\hline
\end{tabular}

Proof. Consider the following quasi-matrix:

The valuations 13 and 14 above show that 1 is invalid, and the valuations 13 and 15 show that 2 is invalid. The remaining cases (De Morgan laws) are left to the reader.

Remark 8. The first four rows of the quasi-matrix above display the semantic values of the propositional variables and the negations of propositional variables that occur in the formulas at stake. The 5th and 6th rows are given by clauses v2 and v3 of Definition 3. Note that the semantic value of $\neg p$ is not determined by the value of $p$ : the value of $\neg p$ bifurcates into 0 and 1 below $v(p)=1$ and also below $v(p)=0$. So, being $n$ the number of propositional variables of a given formula, the number of valuations is finite and bounded by $2^{2 n}$. It is intuitively clear that the valuation semantics provides a decision procedure for FDE. A detailed algorithm, however, will be presented elsewhere.

\subsubsection{Some facts about $F D E$}

Fact 9. Modus ponens and the deduction theorem do not hold in FDE for an implication $\alpha \rightarrow \beta$ defined as $\neg \alpha \vee \beta$.

Proof. That disjunctive syllogism does not hold in FDE is shown by the following valuation: $v(\alpha)=1, v(\neg \alpha)=1, v(\beta)=0$. In order to show that the deduction theorem does not hold, suppose $\Gamma, \alpha \vDash \beta$ implies $\Gamma \vDash \neg \alpha \vee \beta$. So, from $\alpha \vDash \alpha$ we would get $\vDash \neg \alpha \vee \alpha$, but the latter is invalid in $F D E$.

\footnotetext{
${ }^{6} \mathrm{~A}$ quasi-matrix is a non-deterministic matrix that represents non-deterministic valuation semantics. The notion of quasi-matrix was introduced by da Costa and Alves in da Costa and Alves (1977), where a valuation semantics was proposed for da Costa's logic $C_{1}$ (in da Costa and Alves (1977 p. 624, Def. 11) a detailed explanation of how to construct a quasi-matrix for $C_{1}$ can be found). See also Loparic (1986, 2010); Loparic and Alves (1979), where decision procedures based on quasi-matrices are provided for da Costa's $C_{\omega}$ and for intuitionistic logic.
} 
Fact 10. Grounding of contradictoriness

A compound formula $\alpha$ is contradictory in a valuation $v$, i.e. $v(\alpha)=1$ and $v(\neg \alpha)=1$, only if at least one propositional letter $p$ that occurs in $\alpha$ is contradictory in $v$.

Proof. Suppose there is a valuation $v$ such that $v(\alpha)=v(\neg \alpha)=1$. We prove that there is at least one propositional letter $p$ in $\alpha$ such that $v(p)=v(\neg p)=1$. If $\alpha=p$, clearly, $v(\alpha)=v(\neg \alpha)=v(p)=v(\neg p)=1$. The remaining cases are proved by induction on the complexity of $\alpha$.

Case 1. $\alpha=\neg \neg \beta$. I.H.: if $v(\beta)=v(\neg \beta)=1$, there is a $p$ in $\beta$ such that $v(p)=v(\neg p)=1$. Suppose $v(\neg \neg \beta)=v(\neg \neg \neg \beta)=1$. So, by Definition $3, v(\beta)=$ $v(\neg \beta)=1$. The result follows by the inductive hypothesis.

Case 2. $\alpha=\beta \wedge \gamma$. I.H.: if $v(\beta)=v(\neg \beta)=1$, there is a $p$ in $\beta$ such that $v(p)=v(\neg p)=1$; mutatis mutandis for $\gamma$. Suppose $v(\beta \wedge \gamma)=v(\neg(\beta \wedge \gamma))=1$. So, by Definition 3, $v(\beta)=v(\gamma)=1$, and either $v(\neg \beta)=1$ or $v(\neg \gamma)=1$. By the inductive hypothesis, there is a $p$ either in $\beta$ or in $\gamma$ such that $v(p)=v(\neg p)=1$. The remaining cases are left to the reader.

Fact 11. Grounding of incompleteness A compound formula $\alpha$ is incomplete in a valuation $v$, i.e. $v(\alpha)=0$ and $v(\neg \alpha)=0$, only if at least one propositional letter $p$ that occurs in $\alpha$ is incomplete in $v$.

Proof. Similar to the proof of Fact 10 above.

It is to be noted that the converse of Facts 10 and 11 do not hold: there may be a contradictory (resp. incomplete) atom $p$ in a formula $\alpha$ without $\alpha$ being contradictory (resp. incomplete). Let $\alpha$ be the formula $p \vee q$ and consider the valuation $v$ such that $v(p)=v(\neg p)=1, v(q)=1$ and $v(\neg q)=0$. In this case, $p$ is a contradictory propositional letter, but $p \vee q$ is not contradictory. On the other hand, in the valuation $v(p)=v(\neg p)=0, v(q)=1$ and $v(\neg q)=0, p$ is a incomplete propositional letter, but $p \vee q$ is not incomplete. Both valuations make $v(p \vee q)=1$ and $v(\neg(p \vee q))=0$.

\subsection{Equivalence with Belnap's four-valued and Dunn's re- lational semantics}

The valuation semantics proposed above, as expected, is equivalent both to the two-valued relational semantics proposed by Dunn (1976) and to the four-valued semantics presented by Belnap (1977b) 7

Definition 12. Dunn's relational semantics for FDE

A Dunn-interpretation for FDE is a relation $\rho$ between the set of formulas of $F D E$ and the values $T$ and $F, \rho \subseteq L \times\{T, F\}$, satisfying the following clauses:

\footnotetext{
${ }^{7}$ The literature has a variety of algorithmic procedures that provide translations between finite-valued semantics and valuation semantics. One of them is given in Caleiro, Carnielli, Coniglio, and Marcos (2005). For the ease of the reader, however, we give below a direct proof of the equivalence between FDE-valuations, Dunn's and Belnap's semantics for FDE.
} 
1. $\neg \alpha \rho T$ iff $\alpha \rho F$,

2. $\neg \alpha \rho F$ iff $\alpha \rho T$,

3. $(\alpha \wedge \beta) \rho T$ iff $\alpha \rho T$ and $\beta \rho T$,

4. $(\alpha \vee \beta) \rho T$ iff $\alpha \rho T$ or $\beta \rho T$,

5. $(\alpha \wedge \beta) \rho F$ iff $\alpha \rho F$ or $\beta \rho F$,

6. $(\alpha \vee \beta) \rho F$ iff $\alpha \rho F$ and $\beta \rho F$.

Definition 13. A formula $\alpha$ is a Dunn semantic consequence of $\Gamma, \Gamma \vDash_{D} \alpha$, iff for all Dunn-interpretations $\rho$, if $\beta \rho T$ for all $\beta \in \Gamma$, then $\alpha \rho T$.

Definition 14. Belnap's four-valued semantics for FDE A four-valued interpretation for $F D E$ is a function $v_{B}$ from the set of formulas of $F D E$ to the semantic values $\{T, F, B, N\}$ satisfying the following matrices:

\begin{tabular}{c|c}
$\alpha$ & $\neg \alpha$ \\
\hline$T$ & $F$ \\
\hline$F$ & $T$ \\
\hline$B$ & $B$ \\
\hline$N$ & $N$
\end{tabular}

\begin{tabular}{c|c|c|c|c}
$\alpha \wedge \beta$ & $T$ & $F$ & $B$ & $N$ \\
\hline$T$ & $T$ & $F$ & $B$ & $N$ \\
\hline$F$ & $F$ & $F$ & $F$ & $F$ \\
\hline$B$ & $B$ & $F$ & $B$ & $F$ \\
\hline$N$ & $N$ & $F$ & $F$ & $N$
\end{tabular}

\begin{tabular}{c|c|c|c|c}
$\alpha \vee \beta$ & $T$ & $F$ & $B$ & $N$ \\
\hline$T$ & $T$ & $T$ & $T$ & $T$ \\
\hline$F$ & $T$ & $F$ & $B$ & $N$ \\
\hline$B$ & $T$ & $B$ & $B$ & $T$ \\
\hline$N$ & $T$ & $N$ & $T$ & $N$
\end{tabular}

Definition 15. Let $\boldsymbol{D}=\{T, B\}$ be the set of designated values of Belnap's four-valued semantics. A formula $\alpha$ is a four-valued semantic consequence of $\Gamma, \Gamma \vDash_{B} \alpha$, iff for all four-valued interpretations $v_{B}$, if $v_{B}(\beta) \in \boldsymbol{D}$ for all $\beta \in \Gamma$, then $v_{B}(\alpha) \in \boldsymbol{D}$.

The valuation semantics of Definition 3, Dunn's relational semantics of Definition 12, and Belnap's four-valued semantics of Definition 14 intend to represent four scenarios. Belnap (1977b, p. 11) explains the semantic values $T, F$, $N$, and $B$ with the notion of a computer 'being told', so, these values mean, respectively, 'just told true', 'just told false', 'told neither true nor false', and 'told both true and false' (we return to this point in Section 2.2.1 below). Dunn (1976, p. 156) explains them in terms of subsets of $\{T, F\}$, so a proposition can be related to $\{T\},\{F\}, \varnothing$, and $\{T, F\}$. In Section 2 above we explained these four scenarios in terms of availability of evidence.

Although both the valuation semantics proposed here and Dunn's relational semantics are bi-valued, and end up being equivalent, they have an essential difference: a valuation is a function from the set of formulas to $\{0,1\}$, while a Dunn interpretation is a relation between the set of formulas and $\{T, F\}$. In the latter, a formula can be related simultaneously to both $T$ and $F$, when it is, in the Dunn-Belnap reading, both true and false, or not related to $T$ nor $F$, when it is neither true nor false. But these three semantics, as expected, validate the same inferences, i.e. $\Gamma \vDash_{F D E} \alpha$ iff $\Gamma \vDash_{D} \alpha$ iff $\Gamma \vDash_{B} \alpha$. 
Definition 16. (Dunn interpretation induced by an FDE-valuation) Given a FDE-valuation $v$, we define a Dunn-interpretation $\rho_{v}$, based on $v$, as follows:

$$
\begin{aligned}
& \alpha \rho_{v} T \text { iff } v(\alpha)=1 \\
& \alpha \neg \rho_{v} T \text { iff } v(\alpha)=0 \\
& \alpha \rho_{v} F \text { iff } v(\neg \alpha)=1 \\
& \alpha \neg \rho_{v} F \text { iff } v(\neg \alpha)=0
\end{aligned}
$$

Definition 17. (FDE-valuation induced by a Dunn-interpretation)

Given a Dunn-interpretation $\rho$, we define a FDE-valuation $v_{\rho}$, based on $\rho$, as follows:

$$
\begin{aligned}
& v_{\rho}(\alpha)=1 \text { iff } \alpha \rho T \\
& v_{\rho}(\alpha)=0 \text { iff } \alpha \neg \rho T \\
& v_{\rho}(\neg \alpha)=1 \text { iff } \alpha \rho F \\
& v_{\rho}(\neg \alpha)=0 \quad \text { iff } \alpha \neg \rho F
\end{aligned}
$$

Lemma 18. Given an FDE-valuation $v$, then $\rho_{v}$ is a Dunn-interpretation.

Proof. We have to prove that $\rho_{v}$ is a Dunn's relational semantics as in Definition 12 .

1. $\neg \alpha \rho_{v} T$ iff $v(\neg \alpha)=1$ iff $\alpha \rho_{v} F$

2. $\neg \alpha \rho_{v} F$ iff $v(\neg \neg \alpha)=1$ iff $v(\alpha)=1$ iff $\alpha \rho_{v} T$

3. $(\alpha \wedge \beta) \rho_{v} T$ iff $v(\alpha \wedge \beta)=1$ iff $v(\alpha)=1$ and $v(\beta)=1$ iff $\alpha \rho_{v} T$ and $\beta \rho_{v} T$

4. $(\alpha \vee \beta) \rho_{v} T$ iff $v(\alpha \vee \beta)=1$ iff $v(\alpha)=1$ or $v(\beta)=1$ iff $\alpha \rho_{v} T$ or $\beta \rho_{v} T$

5. $(\alpha \wedge \beta) \rho_{v} F$ iff $v(\neg(\alpha \wedge \beta))=1$ iff $v(\neg \alpha)=1$ or $v(\neg \beta)=1$ iff $\alpha \rho_{v} F$ or $\beta \rho_{v} F$

6. $(\alpha \vee \beta) \rho_{v} F$ iff $v(\neg(\alpha \vee \beta))=1$ iff $v(\neg \alpha)=1$ and $v(\neg \beta)=1$ iff $\alpha \rho_{v} F$ and $\beta \rho_{v} F$

Lemma 19. Given a Dunn-intepretation $\rho$, then $v_{\rho}$ is a FDE-valuation.

Proof. We have to prove that $v_{\rho}$ is a $F D E$-valuation as in Definition 3

1. $v_{\rho}(\alpha \wedge \beta)=1$ iff $(\alpha \wedge \beta) \rho T$ iff $\alpha \rho T$ and $\beta \rho T$ iff $v_{\rho}(\alpha)=1$ and $v_{\rho}(\beta)=1$

2. $v_{\rho}(\alpha \vee \beta)=1$ iff $(\alpha \vee \beta) \rho T$ iff $\alpha \rho T$ or $\beta \rho T$ iff $v_{\rho}(\alpha)=1$ or $v_{\rho}(\beta)=1$

3. $v_{\rho}(\neg(\alpha \wedge \beta))=1$ iff $(\alpha \wedge \beta) \rho F$ iff $\alpha \rho F$ or $\beta \rho F$ iff $v_{\rho}(\neg \alpha)=1$ or $v_{\rho}(\neg \beta)=1$ 
4. $v_{\rho}(\neg(\alpha \vee \beta))=1$ iff $(\alpha \vee \beta) \rho F$ iff $\alpha \rho F$ and $\beta \rho F$ iff $v_{\rho}(\neg \alpha)=1$ and $v_{\rho}(\neg \beta)=1$

5. $v_{\rho}(\alpha)=1$ iff $(\alpha) \rho T$ iff $\neg \alpha \rho F$ iff $\neg \neg \alpha \rho T$ iff $v_{\rho}(\neg \neg \alpha)=1$

\section{Lemma 20.}

The valuation semantics (Definition 3) and Dunn-interpretation (Definition 12) are equivalent, that is, given a valuation semantics $v$ there exists a Dunninterpretation $\rho_{v}$ such that

$v_{\rho}(\alpha)=1$ iff $\alpha \rho T$

$v_{\rho}(\alpha)=0$ iff $\alpha \neg \rho T$

$v_{\rho}(\neg \alpha)=1$ iff $\alpha \rho F$

$v_{\rho}(\neg \alpha)=0$ iff $\alpha \neg \rho F$

for any proposition $\alpha$; and vice-versa, given a Dunn-interpretation $\rho$, there exists a valuation $v_{\rho}$ such that:

$\alpha \rho_{v} T$ iff $v(\alpha)=1$

$\alpha \neg \rho_{v} T$ iff $v(\alpha)=0$

$\alpha \rho_{v} F$ iff $v(\neg \alpha)=1$

$\alpha \neg \rho_{v} F$ iff $v(\neg \alpha)=0$

for any proposition $\alpha$.

Proof. Immediate from Lemma 18 and Lemma 19 above.

Lemma 21. The valuation semantics (Definition 3) and Belnap's four-valued semantics for FDE (Definition 14) are equivalent.

Proof. It follows from Lemma 20 and the well-known fact that Dunn's and Belnap's semantics are equivalent.

Theorem 22. The valuation semantics, the Dunn interpretation and the Belnap interpretation define equivalent notions of logical consequence: $\Gamma \vDash_{F D E} \alpha$ iff $\Gamma \vDash_{B} \alpha$ iff $\Gamma \vDash_{D} \alpha$.

Proof. It follows from Lemma 20 and Lemma 21. 


\subsubsection{On paraconsistency, evidence, and information}

$F D E$ is the well-known and widely studied 'useful four-valued logic' proposed by Belnap and Dunn as the underlying logic of an artificial information processor, i.e. a computer, capable of dealing with information received from different sources that are not entirely reliable (cf. Belnap, 1977a b; Dunn, 1976). The semantic value Both is intended to represent the circumstance in which there is conflicting information about $\alpha$, i.e. both $\alpha$ and $\neg \alpha$ hold, and None is intended to represent the circumstance in which there is no information at all about $\alpha$, i.e. neither $\alpha$ nor $\neg \alpha$ holds.

When Belnap explains these four values, he talks about a computer 'being told' that a proposition $\alpha$ is true, or false. The computer should be able to compute the values of complex propositions and draw inferences from the received information, but it "can only accept and report information without divesting itself of it" (Belnap 1977b, p. 9). Of course, contradictory information stored in a database should not be taken as true, as Belnap (1977a, p. 47) remarks that

these sentences have truth-values independently of what the computer has been told; but who can gainsay that the computer cannot use the actual truth-value of the sentences in which it is interested? All it can possibly use as a basis for inference is what it knows or believes, i.e., what it has been told.

The computer, when asked, must provide information based only on what it has been told, otherwise "we would have no way of knowing that its data-base harbored contradictory information" (Belnap, 1977b, p. 9) ${ }^{8}$

This notion of 'a computer being told' is clearly weaker than truth, since a computer may be told that $\alpha$ is true even if it is not the case. So, Belnap is not really talking about truth simpliciter. On the other hand, Dunn (1976, p. 157) seems not to be totally comfortable with the interpretation of $F D E$ in terms of the simultaneous truth of $\alpha$ and $\neg \alpha$ :

Do not get me wrong - I am not claiming that there are sentences which are in fact both true and false. I am merely pointing out that there are plenty of situations where we suppose, assert, believe, etc., contradictory sentences to be true, and we therefore need a semantics which expresses the truth conditions of contradictions in terms of the truth values that the ingredient sentences would have to take for the contradictions to be true.

Indeed, we should consider Dunn's relational semantics as a façon de parler, rather than a claim that true contradictions are possible. Obviously, the simultaneous attribution of the semantic value True to a pair of propositions $\alpha$

\footnotetext{
${ }^{8}$ Belnap's approach to the problem is akin to the idea, defended by us in a number of places, that a contradiction $\alpha$ and $\neg \alpha$ can be 'more informative' than a single assertion of $\alpha$, or of $\neg \alpha$, when neither $\alpha$ nor $\neg \alpha$ has been conclusively established. Indeed, in such cases, the contradiction makes it explicit that something is wrong and must be further investigated.
} 
and $\neg \alpha$ is not to be understood as an acceptance of dialetheism. It is worth noting that at the time Belnap's and Dunn's papers were published, although there were already several paraconsistent formal systems available, the conceptual discussion about the nature of contradictions accepted by paraconsistent logics was still in its beginnings. It was a 'lateral issue' that had not yet been brought to the center of debate.

That the four values represented by Belnap-Dunn's semantics correspond to the four scenarios of availability of evidence the logic $B L E$ expresses has been shown in Section 2.2 above. The notions of evidence and information, indeed, are akin to each other, and both are well-suited to a non-dialetheist reading of paraconsistency. Let us take a closer look at these two notions.

In Carnielli and Rodrigues (2017, Section 2) the notion of evidence for a proposition $\alpha$ was explained as 'reasons for believing and/or accepting $\alpha$ '. Evidence, when conclusive, gives support to the truth (or falsity) of $\alpha$, and thus it has to do with the justification of $\alpha$ (or $\neg \alpha)$. The idea behind the recovery operator $\circ$, introduced in Section 3 below, is that if there is conclusive evidence for the truth, or falsity, of a proposition $\alpha$, then $\alpha$ is subjected to classical logic. But evidence can be non-conclusive, and so there may be conflicting evidence for a proposition $\alpha$. Besides being weaker than truth, evidence does not imply belief: there may be evidence for $\alpha$, an agent may be aware of such evidence but still does not believe in $\alpha$. If there is non-conclusive evidence for $\alpha$, it means that there is some degree of justification for $\alpha$ that, however, is not conclusive and might be wrong. ${ }^{9}$

Dunn 2008, p. 589) explains a 'bare-boned' notion of information as:

what is left from knowledge when you subtract, justification, truth, belief, and any other ingredients such as reliability that relate to justification. Information is, as it were, a mere "idle thought." Oh, one other thing, I want to subtract the thinker. (...) Anyone who has searched for information on the Web does not have to have this concept drummed home. So much of what we find on the Web has no truth or justification, and one would have to be a fool to believe it (...) [Information] is something like a Fregean "thought," i.e., the "content" of a belief that is equally shared by a doubt, a concern, a wish, etc.

Information, so understood, is what is expresses by a proposition, indeed similar to a Fregean thought but without its platonic ingredient. It is objective, does not imply belief, does not need to be true. The difference between this bare-boned notion of information and the notion of non-conclusive evidence is that the latter has an epistemic ingredient that is lacking by the former. So, we can characterize non-conclusive evidence as bare-boned information plus a justification that might be wrong. Indeed, situations in which we have something that may be or may be not a justification for some proposition $\alpha$ are quite common, and there is nothing

${ }^{9}$ This notion of evidence is in line with the discussion carried out in Achinstein (2010a b ) Kelly (2014). 
wrong in saying that evidence, conclusive or non-conclusive, is still information: a proposition $\alpha$ is information, as well as the claim that $\alpha$ has been established as true. The notion of information is thus more general than evidence. It is not surprising, therefore, that both $B L E$ and $F D E$ are suitable to a non-dialetheist interpretation in terms of evidence and information.

\section{Extending FDE to a logic of evidence and truth}

$F D E$ will now be extended to the logic $L E T_{F}$, in a similar way to what was done with $B L E$ obtaining $L E T_{J}$ in Carnielli and Rodrigues (2017). Both $L E T_{J}$ and $L E T_{F}$ are Logics of Formal Inconsistency and Undeterminedness (LFIUs) (cf. Carnielli and Rodrigues, 2017, Carnielli, Coniglio, and Rodrigues, 2019. Marcos, 2005). In LFIs,

$$
\alpha, \neg \alpha \Vdash \beta \text {, while } \circ \alpha, \alpha, \neg \alpha \vdash \beta,
$$

and in $L F U \mathrm{~s}$,

$$
\vdash \alpha \vee \neg \alpha \text {, while } \circ \alpha \vdash \alpha \vee \neg \alpha 10
$$

When $\circ \alpha$ holds, and so excluded middle and explosion are valid, we say that $\alpha$ is classical. For this reason, in $L F I U \mathrm{~s}$, like the logics $L E T_{J}$ and $L E T_{F}$, we say that $\circ$ is a classicality operator.

Like $B L E$, the logic $F D E$, interpreted from the viewpoint of preservation of evidence, is not able to express preservation of truth. Indeed, none of the semantics presented for FDE in Section 2.1 can distinguish a context (i) where there there is non-conclusive evidence for $\alpha$, so $\alpha$ has not been established as true, but no evidence for $\neg \alpha$. from another context (ii) where there is conclusive evidence for $\alpha$ and so $\alpha$ has been established as true, and $\neg \alpha$ does not hold. In both (i) and (ii), $\alpha$ and $\neg \alpha$ receive respectively the values 1 and 0 by the valuation semantics (Definition 3), or the values $T$ and $F$ by the Belnap's four valued semantics (Definition 14), and so we cannot distinguish between (i) and (ii). The logic $L E T_{F}$, on the other hand, is able to distinguish these contexts.

Definition 23. The Logic of Evidence and Truth based on FDE (LET $\left.T_{F}\right)$ Let $L_{2}$ be a language with a denumerable set of sentential letters $\left\{p_{1}, p_{2}, p_{3}, \ldots\right\}$, the set of connectives $\{\circ, \bullet, \neg, \wedge, \vee$,$\} and parentheses. The set of formulas of L_{2}$ is obtained recursively in the usual way. The logic $L E T_{F}$ is defined over the language $L_{2}$ by adding the following rules to the natural deduction system of FDE (Definition 1):

${ }^{10}$ Definitions of Logics of Formal Inconsistency and Undeterminedness can be found in Carnielli, Coniglio, and Rodrigues (2019) (Defs. 9 and 11). Note that the notion of incompleteness in the interpretation of $F D E$ in terms of evidence/information (e.g. Fact 11) is analogous to the notion of undeterminedness in LFUs. Actually, in our view, except for the same acronym of $L F I \mathrm{~s}, L F U$ s could well be called Logics of Formal Incompleteness. The name $L F U$ was established in Marcos (2005) and adopted in Carnielli and Rodrigues (2017) and Carnielli, Coniglio, and Rodrigues (2019). 


$$
\begin{array}{lc}
\frac{\circ \alpha \bullet \alpha}{\beta} \text { Cons } & \frac{\circ \alpha \vee \bullet \alpha}{\circ \alpha o m p} \\
\frac{\circ \alpha \quad \alpha \quad \neg \alpha}{\beta} E X P^{\circ} & \frac{\circ \alpha}{\alpha \vee \neg \alpha} P E M^{\circ}
\end{array}
$$

$A$ deduction of $\alpha$ from a set of premises $\Gamma$ in $L E T_{F}, \Gamma \vdash_{L E T_{F}} \alpha$, is defined as follows: there is a derivation with conclusion $\alpha$ and all uncancelled hypotheses in $\Gamma$. The definition of a derivation is the usual one for natural deduction systems (see e.g. van Dalen (2008, pp. 35-36)).

Theorem 24. The following properties hold for $L E T_{F}$ :

1. Reflexivity: if $\alpha \in \Gamma$, then $\Gamma \vdash{ }_{L E T_{F}} \alpha$;

2. Monotonicity: if $\Gamma \vdash_{L E T_{F}} \beta$, then $\Gamma, \alpha \vdash_{L E T_{F}} \beta$, for any $\alpha$;

3. Transitivity (cut): if $\Delta \vdash_{L E T_{F}} \alpha$ and $\Gamma, \alpha \vdash_{L E T_{F}} \beta$, then $\Delta, \Gamma \vdash_{L E T_{F}} \beta$;

4. Compactness: if $\Gamma \vdash_{L E T_{F}} \alpha$, then there is $\Delta \subseteq \Gamma, \Delta$ finite such that $\Delta \vdash{ }_{L E T_{F}} \alpha$.

Proof. Straightforward, from the definition of a deduction of $\alpha$ from premises in $\Gamma$ in $L E T_{F}$.

Fact 25. The following rules hold in $L E T_{F}$ :

$$
\frac{\alpha \neg \alpha}{\bullet \alpha} \bullet R 1 \quad \frac{}{\alpha \vee \neg \alpha \vee \bullet \alpha} \bullet R 2
$$

Proof. We prove $\bullet R 1$. The proof of $\bullet R 2$ is left to the reader.

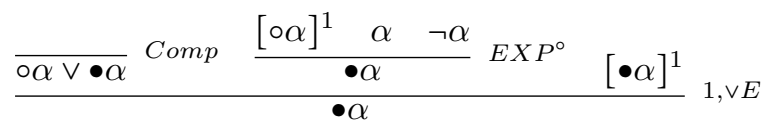

\subsection{On the connectives $\circ$ and •}

The rules $P E M^{\circ}$ and $E X P^{\circ}$ recover classical logic for propositions in the scope of o (this claim will be made precise by Fact 31 below). As well as $L E T_{J}, L E T_{F}$ is suitable to an intuitive reading in terms of different contexts concerned with preservation of evidence and preservation of truth. But unlike $L E T_{J}, L E T_{F}$ has a non-classicality operator $\bullet$, dual to the classicality operator $\circ$. This duality is made clear by the rules above (Fact 25): $R 1$ is the dual of $E X P^{\circ}$, and $R 2$ is the dual of $P E M^{\circ 11}$. While $\circ \alpha$ implies a classical behavior for $\alpha$, a non-classical

\footnotetext{
${ }^{11}$ Actually, different versions of $L E T_{F}$ can be obtained by adding to $F D E$, besides Cons and Comp, the following pair of rules: $P E M^{\circ}$ and $E X P^{\circ} ; \bullet R 1$ and $\bullet R 2 ; P E M^{\circ}$ and $\bullet R 1$; $E X P^{\circ}$ and $\bullet R 2$. Notice that the rules $E X P^{\circ}$ and $\bullet R 2$ are dual, as well as $P E M^{\circ}$ and $\bullet R 1$ (cf. Carnielli et al. 2019).
} 
behavior of $\alpha$ implies $\bullet \alpha$. Notice that: (i) $\circ \alpha$ does not imply $\alpha$, rather, it implies that one and at most one between $\alpha$ and $\neg \alpha$ holds; (ii) $\bullet \alpha$ does not imply that $\alpha$ and $\neg \alpha$ hold; indeed, according to $R 2$, if both $\alpha$ and $\neg \alpha$ do not hold, $\bullet \alpha$ holds.

Strictly speaking, $\circ \alpha$ recovers classical logic for $\alpha$. The intended interpretation of $\circ \alpha$ is that there is conclusive evidence for $\alpha$ or $\neg \alpha$, and so the truth-value of $\alpha$ is conclusively established as true or false. On the other hand, if evidence for $\alpha$ is non-conclusive, or it is contradictory, or there is no evidence at all, then - $\alpha$ holds. The rule Cons prohibits the circumstance such that there is and there is not conclusive evidence for $\alpha$, while Comp expresses the fact that either there is or there is not conclusive evidence for $\alpha$.

Since $L E T_{F}$ distinguishes conclusive from non-conclusive evidence, it is able to express the following six scenarios ${ }^{12}$

When $\bullet \alpha$ holds, four scenarios of non-conclusive evidence can be expressed:

1. Only evidence that $\alpha$ is true: $\alpha$ holds, $\neg \alpha$ does not hold.

2. Only evidence that $\alpha$ is false: $\neg \alpha$ holds, $\alpha$ does not hold.

3. No evidence at all: both $\alpha$ and $\neg \alpha$ do not hold.

4. Conflicting evidence: both $\alpha$ and $\neg \alpha$ hold.

When $\circ \alpha$ holds, two scenarios can be expressed, tantamount to classical truth and falsity:

5. Conclusive evidence for $\alpha: \alpha$ is true ( $\circ \alpha \wedge \alpha$ holds).

6. Conclusive evidence for $\neg \alpha: \neg \alpha$ is true ( $\circ \alpha \wedge \neg \alpha$ holds).

Of course, a scenario with conclusive evidence for both $\alpha$ and $\neg \alpha$ is not allowed, since it would imply that $\alpha$ is true and false simultaneously. Indeed, if classical logic holds for $\alpha$, it cannot be that there is any residual conflicting evidence for $\alpha$ and $\neg \alpha$.

\subsection{Valuation semantics for $L E T_{F}$}

Definition 26. A valuation semantics for $L E T_{F}$ is obtained by adding the following clauses to the valuation semantics of FDE (Definition 3):

v6. $v(\bullet \alpha)=1$ iff $v(\circ \alpha)=0$,

v\%. If $v(\circ \alpha)=1$, then $v(\alpha)=1$ if and only if $v(\neg \alpha)=0$.

${ }^{12}$ In classical logic, ' $\alpha$ holds' means that $\alpha$ is true, while in $F D E$, according to the intended interpretation in terms of evidence, ' $\alpha$ holds' means that there is evidence available for $\alpha$. In $L E T_{F}$, the meaning of ' $\alpha$ holds' depends on the context: if the context is classical, it means that $\alpha$ is true. This is precisely the point of the classicality operator $\circ$. So, two additional scenarios can be expressed, besides the four scenarios of FDE. 
Definition 27. We say that a formula $\alpha$ is a semantical consequence of $\Gamma$, $\Gamma \vDash_{L E T_{F}} \alpha$ iff for every valuation $v$, if $v(\beta)=1$ for all $\beta \in \Gamma$, then $v(\alpha)=1$.

The valuation semantics given above in Definition 26 is sound, complete, and provides a decision procedure for $L E T_{F}$. From now on, when there is no risk of ambiguity, we will just write $\vdash$ and $\vDash$ in the place of $\vdash_{L E T_{F}}$ and $\vDash_{L E T_{F}}$.

Theorem 28. Soundness and completeness of $L E T_{F}$ w.r.t. the valuation semantics: $\Gamma \vDash \alpha$ iff $\Gamma \vdash \alpha$.

Proof. In order to prove completeness, the proof of Theorem 6 has to be extended to include clauses $6^{\prime}$ and $7^{\prime}$ below:

$v 6^{\prime} . \circ \alpha \in \Delta$ iff $\bullet \alpha \notin \Delta$,

$v^{\prime 7^{\prime}} . \circ \alpha \in \Delta$ implies $\neg \alpha \in \Delta$ iff $\alpha \notin \Delta$.

For soundness, it can be shown that rules Cons, Comp, EXP $P^{\circ}$, and $P E M^{\circ}$ are sound with respect to clauses 6 and 7 of Definition 26 above. Details are left to the reader.

The quasi-matrix below displays the behavior of the connectives $\circ$ and $\bullet$ in $L E T_{F}$.

\begin{tabular}{|c|c|c|c|c|c|c|}
\hline$p$ & \multicolumn{3}{|c|}{0} & \multicolumn{3}{c|}{1} \\
\hline$\neg p$ & 0 & \multicolumn{2}{|c|}{1} & \multicolumn{2}{|c|}{0} & 1 \\
\hline$\circ p$ & 0 & 1 & 0 & 1 & 0 & 0 \\
\hline$\bullet p$ & 1 & 0 & 1 & 0 & 1 & 1 \\
\hline valuation & 1 & 2 & 3 & 4 & 5 & 6 \\
\hline
\end{tabular}

The first two lines display the possible values of $p$ and $\neg p$. The connectives $\circ$ and $\bullet$ are primitive and unary, but the semantic values of $o p$ and $\bullet p$ depend (non-deterministically) on the semantic values of $p$ and $\neg p$. When $v(p)=1$ and $v(\neg p)=0, v(p)=0$ and $v(\neg p)=1$, the value of $\circ p$ and $\bullet p$ bifurcates into 0 and 1. This expresses the fact that op is undetermined in $L E T_{F}$ when $v(p) \neq v(\neg p)$, as explained in page 14 above. In terms of evidence, valuations $v_{1}$ and $v_{6}$ show, respectively, that no evidence at all, as well as conflicting evidence, implies $v(\bullet p)=1$ and $v(\circ p)=0$. But if only one holds among $p$ and $\neg p$ (valuations $v_{2}$ to $\left.v_{5}\right)$, then $v(\bullet p)$ and $v(\circ p)$ are left undetermined. The rationale of this is that in order to say that $p$ is true, or false, only the information that there is evidence for the truth, or for the falsity, of $p$ is not enough. Something else is needed, namely, the information that such evidence is conclusive ${ }^{13}$

In Example 29 below we illustrate how quasi-matrices work in $L E T_{F}$.

\footnotetext{
${ }^{13}$ Note that valuations express evidence available from a purely qualitative point of view. An analogy with analytical chemistry at this point may be illuminating. Qualitative analysis is concerned with whether or not some sample contains a given substance, while quantitative analysis asks how much of a substance is contained in a sample. Analogously, the valuation semantics represents only that there is or there is not positive and negative evidence available for $\alpha$, while the probabilistic semantics, presented in Section 4 below, intends to express the amount of such evidence.
} 
Example 29. In $L E T_{F}$ :

$$
\begin{aligned}
& \text { 1. } p \vee \neg p \not \circ p \\
& \text { 2. } \bullet p \vDash p \wedge \neg p \\
& \text { 3. } \circ p, p, \neg p \vee q \vDash q ; \\
& \text { 4. } \circ p, p, \neg(p \wedge q) \vDash \neg q \text {; }
\end{aligned}
$$

\begin{tabular}{|c|c|c|c|c|c|c|c|c|c|c|c|c|}
\hline$p$ & \multicolumn{12}{|c|}{0} \\
\hline$\neg p$ & \multicolumn{4}{|c|}{0} & \multicolumn{8}{|c|}{1} \\
\hline$q$ & \multicolumn{2}{|c|}{0} & \multicolumn{2}{|c|}{1} & \multicolumn{4}{|c|}{0} & \multicolumn{4}{|c|}{1} \\
\hline$\neg q$ & 0 & 1 & 0 & 1 & \multicolumn{2}{|c|}{0} & \multicolumn{2}{|c|}{1} & \multicolumn{2}{|c|}{0} & \multicolumn{2}{|c|}{1} \\
\hline$\neg p \vee q$ & 0 & 0 & 1 & 1 & \multicolumn{2}{|c|}{1} & \multicolumn{2}{|c|}{1} & \multicolumn{2}{|c|}{1} & \multicolumn{2}{|c|}{1} \\
\hline$\neg(p \wedge q)$ & 0 & 1 & 0 & 1 & \multicolumn{2}{|c|}{1} & & & \multicolumn{2}{|c|}{1} & \multicolumn{2}{|c|}{1} \\
\hline$o p$ & 0 & 0 & 0 & 0 & 0 & 1 & 0 & 1 & 0 & 1 & 0 & 1 \\
\hline$\bullet p$ & 1 & 1 & 1 & 1 & 1 & 0 & 1 & 0 & 1 & 0 & 1 & 0 \\
\hline valuation & 1 & 2 & 3 & 4 & 5 & 6 & 7 & 8 & 9 & 10 & 11 & 12 \\
\hline
\end{tabular}

\begin{tabular}{|c|c|c|c|c|c|c|c|c|c|c|c|c|}
\hline$p$ & \multicolumn{12}{|c|}{1} \\
\hline$\neg p$ & \multicolumn{8}{|c|}{0} & \multicolumn{4}{|c|}{1} \\
\hline$q$ & \multicolumn{4}{|c|}{0} & \multicolumn{4}{|c|}{1} & \multicolumn{2}{|c|}{0} & \multicolumn{2}{|c|}{1} \\
\hline$\neg q$ & \multicolumn{2}{|c|}{0} & \multicolumn{2}{|c|}{1} & \multicolumn{2}{|c|}{0} & \multicolumn{2}{|c|}{1} & 0 & 1 & 0 & 1 \\
\hline$\neg p \vee q$ & \multicolumn{2}{|c|}{0} & \multicolumn{2}{|c|}{0} & \multicolumn{2}{|c|}{1} & \multicolumn{2}{|c|}{1} & 1 & 1 & 1 & 1 \\
\hline$\neg(p \wedge q)$ & \multicolumn{2}{|c|}{0} & \multicolumn{2}{|c|}{1} & \multicolumn{2}{|c|}{0} & \multicolumn{2}{|c|}{1} & 1 & 1 & 1 & 1 \\
\hline$\circ p$ & 0 & 1 & 0 & 1 & 0 & 1 & 0 & 1 & 0 & 0 & 0 & 0 \\
\hline$\bullet p$ & 1 & 0 & 1 & 0 & 1 & 0 & 1 & 0 & 1 & 1 & 1 & 1 \\
\hline valuation & 13 & 14 & 15 & 16 & 17 & 18 & 19 & 20 & 21 & 22 & 23 & 24 \\
\hline
\end{tabular}

Proof. Consider the following quasi-matrix (divided into two parts):

Item 1: since $v_{24}(p)=v_{24}(\neg p)=1, v_{24}(p \vee \neg p)=1$, but $v_{24}(\circ p)=0$. Item 2 : $v_{1}(\bullet p)=1$, but $v_{1}(p \wedge \neg p)=0$, since $v_{1}(p)=v_{1}(\neg p)=0$. For items 3 and 4 , it is easy to check that there is no valuation $v$ such that the premises receive the value 1 but the conclusion receives 0 in $v$ (compare with items 1 and 2 of Fact 7).

Remark 30. The 7th row of the quasi-matrix above is given by clause $v 7$ and the 8th by clause v6 of Definition 26. A quasi-matrix for $L E T_{F}$ is finite, and similarly to FDE (see Remark 8), it is intuitively clear that the valuation semantics provides a decision procedure for $L E T_{F}$. A detailed algorithm will be presented elsewhere.

\subsection{Some facts about $L E T_{F}$}

Fact 31 below shows how the operator o recovers classical logic in $L E T_{F}$. 
Fact 31. Recovering classical logic in $L E T_{F}$ Suppose $\circ \neg^{n_{1}} \alpha_{1}, \circ \neg^{n_{2}} \alpha_{2}, \ldots, \circ \neg^{n_{m}} \alpha_{m}$ hold, for $n_{i} \geq 0$ (where, $\neg^{n_{i}}, n_{i} \geq 0$, represents $n_{i}$ iterations of negations of the formula $\left.\alpha_{i}\right)$. Then, for any formula $\beta$ formed with $\alpha_{1}, \alpha_{2}, \ldots, \alpha_{m}$ over $\{\wedge, \vee, \neg\}, \beta$ behaves classically.

Proof.

First, we show that for any value of $n_{i} \geq 0, \circ \neg^{n_{i}} \alpha_{i} \vdash \alpha_{i} \vee \neg \alpha_{i}$ and $\circ \neg^{n_{i}} \alpha_{i}, \alpha_{i} \wedge$ $\neg \alpha_{i} \vdash \gamma$, for any $\gamma-$ i.e. excluded middle and explosion hold for $\alpha_{i}$.

Suppose $\circ \neg^{n_{i}} \alpha_{i}$ holds. So, $\neg \neg^{n_{i}} \alpha_{i} \vee \neg \neg^{n_{i}} \alpha_{i}$ and $\left.\neg\right\urcorner^{n_{i}} \alpha_{i} \wedge \neg \neg^{n_{i}} \alpha_{i} \vdash \gamma$ hold. If $n_{i}$ is even, $\neg^{n_{i}} \alpha_{i} \dashv \vdash \alpha_{i}$, and if $n_{i}$ is odd, $\neg^{n_{i}} \alpha_{i} \dashv \vdash \neg \alpha_{i}$. So, it is easily proved that $\neg^{n_{i}} \alpha_{i} \vee \neg \neg^{n_{i}} \alpha_{i} \vdash \alpha_{i} \vee \neg \alpha_{i}$. Since we have that $\circ^{n^{n_{i}}} \alpha_{i} \vdash \neg^{n_{i}} \alpha_{i} \vee \neg \neg^{n_{i}} \alpha_{i}$, by transitivity, we get $\circ^{\prime}{ }^{n_{i}} \alpha_{i} \vdash \alpha_{i} \vee \neg \alpha_{i}$. In order to recover explosion, it can be easily proved that $\alpha_{i} \wedge \neg \alpha_{i} \vdash \neg^{n_{i}} \alpha_{i} \wedge \neg \neg^{n_{i}} \alpha_{i}$. Since we have that $\circ \neg^{n_{i}} \alpha_{i}, \neg^{n_{i}} \alpha_{i} \wedge$ $\neg \neg^{n_{i}} \alpha_{i} \vdash \gamma$, by transitivity, we get $\circ \neg^{n_{i}} \alpha_{i}, \alpha_{i} \wedge \neg \alpha_{i} \vdash \gamma$.

Remember that full classical logic can be obtained by adding explosion and excluded middle to the introduction and elimination rules of $\wedge$ and $\vee, \alpha_{1} \rightarrow \alpha_{2}$ being defined as $\neg \alpha_{1} \vee \alpha_{2}$. Now, in order to prove the result, it is enough to show that for any formula $\beta$ formed with $\alpha_{1}, \alpha_{2}, \ldots, \alpha_{m}$ over $\{\wedge, \vee, \neg\}$, if $\circ \neg^{n_{1}} \alpha_{1}, \circ \neg^{n_{2}} \alpha_{2}, \ldots, \circ \neg^{n_{m}} \alpha_{m}$ hold, then $\vdash \beta \vee \neg \beta$ and $\beta, \neg \beta \vdash \gamma$ hold.

Let $\Gamma=\left\{\circ \neg^{n_{1}} \alpha_{1}, \circ \neg^{n_{2}} \alpha_{2}, \ldots, \circ \neg^{n_{m}} \alpha_{m}\right\}$.

If $\beta=\alpha_{i}$, it has been proved above. The remaining cases are proved by induction on the complexity of $\beta$.

Case 1. $\beta=\neg \delta$. I.H. $\Gamma, \delta, \neg \delta \vdash \gamma$ and $\Gamma \vdash \delta \vee \neg \delta$. It can be easily proved that $\Gamma, \neg \delta, \neg \neg \delta \vdash \gamma$ and $\Gamma \vdash \neg \delta \vee \neg \neg \delta$.

Case 2. $\beta=\delta_{1} \wedge \delta_{2}$. I.H. $\Gamma, \delta_{1}, \neg \delta_{1} \vdash \gamma$ and $\Gamma \vdash \delta_{1} \vee \neg \delta_{1}$, mutatis mutandis for $\delta_{2}$. It can be proved that $\Gamma, \delta_{1} \wedge \delta_{2}, \neg\left(\delta_{1} \wedge \delta_{2}\right) \vdash \gamma$ and $\Gamma \vdash\left(\delta_{1} \wedge \delta_{2}\right) \vee \neg\left(\delta_{1} \wedge \delta_{2}\right)$ The remaining cases are left to the reader.

We have seen in Fact 9 that for an implication $\alpha \rightarrow \beta$ defined in FDE as $\neg \alpha \vee \beta$, modus ponens and the deduction theorem do not hold. Both are recovered for the defined implication in $L E T_{F}$ for classical propositions.

Fact 32.

1. In $L E T_{F}$, for classical propositions, modus ponens holds: $\circ \alpha, \alpha, \neg \alpha \vee \beta \vdash \beta$.

Proof.

$\frac{\neg \alpha \vee \beta \frac{\circ \alpha \quad \alpha \quad[\neg \alpha]^{1}}{\beta} E X P^{\circ}[\beta]^{1}}{\beta} 1, \vee E$

2. In $L E T_{F}$, the following form of the deduction theorem holds: $\circ \alpha, \alpha \vdash \beta$ implies $\circ \alpha \vdash \neg \alpha \vee \beta$. 
Proof.

$$
\begin{gathered}
\circ \alpha,[\alpha]^{1} \\
\vdots \\
\frac{\circ{ }^{\alpha \vee \neg \alpha} P E M^{\circ}}{\frac{\dot{\beta}}{\neg \alpha \vee \beta} \vee I \quad \frac{[\neg \alpha]^{1}}{\neg \alpha \vee \beta}} \underset{ }{\neg \alpha}{ }_{1, \vee E}
\end{gathered}
$$

584

Definition 33. Supplementing and complementing negations (Carnielli et al. 2007, pp. 12ff)

1. We say that a unary connective * in a logic $\mathcal{L}$ is a supplementing negation if: (i) for some formula $\alpha, * \alpha$ is not a bottom particle, and (ii) for any $\Gamma, \alpha$ and $\beta: \Gamma, \alpha, * \alpha \vdash_{\mathcal{L}} \beta$.

2. We say that a unary connective * in a logic $\mathcal{L}$ is a complementing negation if: (i) for some formula $\alpha, * \alpha$ is not a top particle;

(ii) for any $\Gamma, \alpha$ and $\beta: \Gamma, \alpha \vdash_{\mathcal{L}} \beta$ and $\Gamma, * \alpha \vdash_{\mathcal{L}} \beta$ implies $\Gamma \vdash_{\mathcal{L}} \beta$.

If $*$ is a complementing negation, for any $\alpha$, at least one between $\alpha$ and $* \alpha$ hold, and if $*$ is a complementing negation, it cannot be that both $\alpha$ and $* \alpha$ hold. Each one expresses one half of classical negation, the former excluded middle, the latter explosion. If a logic $\mathcal{L}$ has a (primitive or defined) negation connective that is both supplementing and complementing, then $\mathcal{L}$ has a classical negation. A complementing negation and a supplementing negation can be defined in $L E T_{F}$.

Definition 34. The following unary connectives can be defined in $L E T_{F}$ :

1. The connective truth: $\oplus \alpha \stackrel{\text { def }}{=} \circ \alpha \wedge \alpha$;

2. The connective falsity: $\sim \alpha \stackrel{\text { def }}{=} \circ \alpha \wedge \neg \alpha$;

3. The connective falsity-excluding: $\ominus \alpha \stackrel{\text { def }}{=} \alpha \vee \alpha$;

4. The connective truth-excluding: $\approx \alpha \stackrel{\text { def }}{=} \alpha \vee \neg \alpha$.

The tables are the following:

\begin{tabular}{|c|c|c|c|c|c|c|}
\hline$\alpha$ & \multicolumn{3}{|c|}{0} & \multicolumn{3}{|c|}{1} \\
\hline$\neg \alpha$ & 0 & \multicolumn{2}{|c|}{1} & \multicolumn{2}{|c|}{0} & 1 \\
\hline$\circ \alpha$ & 0 & 0 & 1 & 0 & 1 & 0 \\
\hline$\bullet \alpha$ & 1 & 1 & 0 & 1 & 0 & 1 \\
\hline$\oplus \alpha$ & 0 & 0 & 0 & 0 & 1 & 0 \\
\hline$\sim \alpha$ & 0 & 0 & 1 & 0 & 0 & 0 \\
\hline$\ominus \alpha$ & 1 & 1 & 0 & 1 & 1 & 1 \\
\hline$\approx \alpha$ & 1 & 1 & 1 & 1 & 0 & 1 \\
\hline
\end{tabular}


These connectives have been named for the following reasons. According to the proposed interpretation: (1) $\circ \alpha \wedge \alpha$ means that there is conclusive evidence for $\alpha$, and so $\alpha$ is true $(\oplus \alpha)$; (2) $\circ \wedge \wedge \neg$ means that there is conclusive evidence for the falsity of $\alpha$, and so $\alpha$ is false $(\sim \alpha)$; (3) $\bullet \alpha \vee \alpha$ means that there is no conclusive evidence for $\alpha$, or $\alpha$ holds, and so it excludes the falsity of $\alpha(\ominus \alpha)$; (4) $\bullet \alpha \vee \neg \alpha$ means that there is no conclusive evidence for $\alpha$, or $\neg \alpha$ holds, and so it excludes the truth of $\alpha(\approx \alpha)$. It is also clear from the table above and Definition 33 that $\sim \alpha$ is a supplementing negation (if $v(\alpha)=1, v(\sim \alpha)=0$, they cannot be both 1 ), while $\approx \alpha$ is a complementing negation (if $v(\alpha)=0, v(\approx \alpha)=1$, they cannot be both 0$){ }^{14}$ We conjecture that no classical negation can be defined in $L E T_{F}{ }^{15}$

These four connectives enjoy some interesting logical relations w.r.t. each other that can be displayed by a square of oppositions:

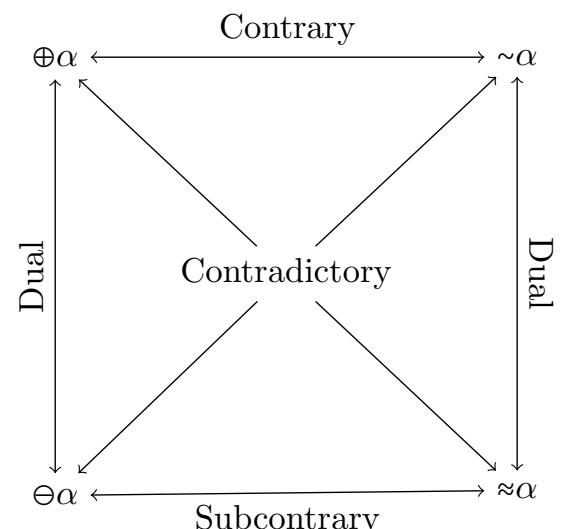

$\sim \alpha$ and $\oplus \alpha$ are contrary propositions (i.e., they can both be false, but they cannot both be simultaneously true); $\approx \alpha$ and $\ominus \alpha$ are subcontrary propositions (i.e., they can both be true, but they cannot both be simultaneously false); $\oplus \alpha$ (resp. $\sim \alpha$ ) is the dual of $\ominus \alpha$ (resp. $\approx \alpha$ ); $\oplus \alpha$ (resp. $\sim \alpha$ ) is the contradictory of $\approx \alpha$ (resp. $\ominus$ ). Notice that in $L E T_{F}, \circ$ is the dual of $\bullet$, and $\neg$ is the dual of itself (on duality between non-deterministic connectives in Logics of Formal Inconsistency and Undeterminedness, see Carnielli et al. (2019)).

\section{Fact 35 .}

1. $\circ \alpha \wedge \alpha \wedge \neg \alpha, \circ \alpha \wedge \bullet \alpha, \oplus \alpha \wedge \sim \alpha, \oplus \alpha \wedge \approx \alpha$, and $\ominus \alpha \wedge \sim \alpha$ are bottom particles in $L E T_{F}$.

\footnotetext{
${ }^{14}$ Although $\sim$ is explosive, it is not a classical negation, since $\alpha \vee \sim \alpha$ does not hold, which is shown by the valuation $v(\alpha)=v(\neg \alpha)=v(\circ \alpha)=0$, and although $\alpha \vee \approx \alpha$ holds, $\approx$ is also not a classical negation, since $\alpha, \approx \alpha \vdash \beta$ does not hold, which is shown by the valuation $v(\alpha)=v(\neg \alpha)=v(\bullet \alpha)=1$.

${ }^{15}$ One possibility for proving that classical negation is not definable in $L E T_{F}$ is to adapt the methods of Lahav, Marcos, and Zohar (2016), although they are devoted to non-classical negations from a modal viewpoint. We have been unable however, to find a convincing argument in this direction.
} 
2. $\sim$ is a supplementing negation in $L E T_{F}$.

3. $\approx$ is a complementing negation in $L E T_{F}$.

Proof. In a few steps from the rules Cons, EXP $P^{\circ}$ and $\bullet R 2$.

Theorem 36. The following propositions are theorems of $L E T_{F}$ :

1. $\circ \alpha \vee \bullet \alpha$

2. $\alpha \vee \neg \alpha \vee \bullet \alpha$

3. $(\bullet \alpha \wedge \alpha) \vee(\bullet \alpha \wedge \neg \alpha) \vee \bullet \alpha \vee(\bullet \alpha \wedge \alpha \wedge \neg \alpha) \vee(\circ \alpha \wedge \alpha) \vee(\circ \alpha \wedge \neg \alpha)$

4. $\alpha \vee \neg \alpha \vee \ominus \alpha$

5. $\alpha \vee \neg \alpha \vee \approx \alpha$

6. $\oplus \alpha \vee \sim \alpha \vee \bullet \alpha$

Proof. Items 1 and 2 follow from the rules Comp and $R 2$. To prove 3, from $\circ \alpha \vdash \alpha \vee \neg \alpha$, we obtain $\circ \alpha \vdash(\circ \alpha \wedge \alpha) \vee(\circ \alpha \wedge \neg \alpha)$, and so $\circ \alpha \vdash(\bullet \alpha \wedge \alpha) \vee$ $(\bullet \alpha \wedge \neg \alpha) \vee \bullet \alpha \vee(\bullet \alpha \wedge \alpha \wedge \neg \alpha) \vee(\circ \alpha \wedge \alpha) \vee(\circ \alpha \wedge \neg \alpha)$. On the other hand, $\bullet \alpha \vdash(\bullet \alpha \wedge \alpha) \vee(\bullet \alpha \wedge \neg \alpha) \vee \bullet \alpha \vee(\bullet \alpha \wedge \alpha \wedge \neg \alpha) \vee(\circ \alpha \wedge \alpha) \vee(\circ \alpha \wedge \neg \alpha)$ holds. Now, use 1 and $\vee E$. The proofs of 4,5 , and 6 are left to the reader. Notice that item 3 corresponds to the six scenarios presented in Section 3.1.

\section{Probabilistic semantics for $L E T_{F}$}

We now present a probabilistic semantics for $L E T_{F}$ and $F D E$.

Definition 37. Given a logic $\mathcal{L}$, with a derivability relation $\vdash$ and a language $L$, a probability distribution for $\mathcal{L}$ is a real-valued function $P: L \rightarrow \mathbb{R}$ satisfying the following conditions:

1. Non-negativity: $0 \leq P(\alpha) \leq 1$ for all $\alpha \in L$;

2. Tautologicity: If $\vdash \alpha$, then $P(\alpha)=1$;

3. Anti-Tautologicity: If $\alpha=1$, then $P(\alpha)=0$;

4. Comparison: If $\alpha \vdash \beta$, then $P(\alpha) \leq P(\beta)$;

5. Finite additivity: $P(\alpha \vee \beta)=P(\alpha)+P(\beta)-P(\alpha \wedge \beta)$.

The clauses above can be regarded as meta-axioms that define probability functions for an appropriate logic $\mathcal{L}$ just by taking $\vdash$ as the derivability relation of $\mathcal{L}$, and so the notion of probability can be regarded as logic-dependent. These clauses define probability functions for both $F D E$ and $L E T_{F}$ just by employing 
respectively $\vdash_{F D E}$ and $\vdash_{L E T_{F}} \sqrt{16}$ From now on, we will concentrate on $L E T_{F}$, but it should be clear that the meta-axioms 1, 4 and 5 above define probability distributions for FDE as well.

\section{Definition 38. $L E T_{F}$-probability distribution}

Let $\Sigma=\left\{\alpha_{1}, \cdots, \alpha_{n}, \cdots\right\}$ be a (finite or infinite) collection of propositions in the language $L_{2}$ of $L E T_{F}$. A $L E T_{F}$-probability distribution over $\Sigma$ is an assignment of probability values $P$ to the elements of $\Sigma$ that can be extended to a full probability function $P: L_{2} \rightarrow \mathbb{R}$ according to Definition 37 .

It is a common view that the classical truth-values true (1) and false (0) can be identified with the endpoints of probabilities in the unit interval $[0,1]$. On the other hand, interpretations $v: L \rightarrow\{0,1\}$ of a formal language $L$ can be regarded as degenerate probability functions $P: L \rightarrow[0,1]$. The class of logics that make possible such an identification can be seen as a special case of probability logic. The standard view, however, is rather the opposite: it claims that probability logic presupposes, and so it depends on, classical logic ${ }^{17}$ But the connection between logic and probability theory is far from being restricted to classical logic. The fact that probability distributions can be defined based on a non-classical consequence relation, in our view, makes clear that the relation between logic and probability goes beyond the realm of classical logic.

\subsection{Conditional probability}

The notion of conditional probability of $\alpha$ given $\beta$ is defined as usual, for $P(\beta) \neq 0$ :

$$
P(\alpha / \beta)=\frac{P(\alpha \wedge \beta)}{P(\beta)}
$$

In terms of evidence, a statement $P(\alpha / \beta)$ is to be read as a measure of how much the evidence available for $\beta$ affects the evidence for $\alpha$.

Some useful theorems of conditional probability of $L E T_{F}$-distributions are the following, with the caveat that $P(\beta) \neq 0$ in all cases where $P(\alpha / \beta)$ is mentioned:

\footnotetext{
${ }^{16}$ Probability functions have been defined in this way for classical logic, for intuitionistic logic without implication in Weatherson (2003), and for the paraconsistent logics Ci and Cie in Bueno-Soler and Carnielli (2016, 2017).

${ }^{17}$ In a recent article, Demey et al. (2013) claim that "probability theory presupposes and extends classical logic", and leave aside all the attempts to combine probability theory with non-classical logics. These attempts, however, not only do exist, but have also been successful in combining probability theory with non-classical approaches to logical consequence. We think Demey et al. are mistaken, not only because they ignore non-classical approaches to probability logic, but also because they underestimate the view according to which classical and some non-classical logics can be seen as special cases of probability logic. It is worth noting that attempts to put together probability theory and non-classical logics can be traced back to Lukasiewicz (1913) and Tarski (1935).
} 
Theorem 39.

The following properties hold when the probabilities in the denominators are different from 0.

1. $P\left(\alpha_{1} \wedge \ldots \wedge \alpha_{n}\right)=P\left(\alpha_{1} / \alpha_{2} \wedge \ldots \wedge \alpha_{n}\right) \ldots P\left(\alpha_{n-1} / \alpha_{n}\right) P\left(\alpha_{n}\right)$ (Chain Rule).

2. $P(\alpha / \beta \wedge \gamma)=\frac{P(\alpha / \gamma) \cdot P(\beta / \alpha \wedge \gamma)}{P(\beta / \gamma)}$.

3. $P(\alpha \wedge \beta / \gamma)=P(\alpha / \gamma) \cdot P(\beta / \alpha \wedge \gamma)=P(\beta / \gamma) \cdot P(\alpha / \beta \wedge \gamma)$.

4. $P(\alpha \vee \beta / \gamma)=P(\alpha / \gamma)+P(\beta / \gamma)-P(\alpha \wedge \beta / \gamma)$.

5. $P(\alpha \vee \beta / \gamma)=P(\alpha / \gamma)+P(\beta / \gamma)$ if $\alpha$ and $\beta$ are logically incompatible, i.e., $\alpha \wedge \beta$ act as a $\perp$ (see Section 4.2).

6. $P(\alpha / \beta)+P(\neg \alpha / \beta)-P(\bullet \alpha / \beta) \leq P(\alpha \vee \neg \alpha / \beta)$.

7. If $P(\circ \alpha)=1$, or equivalently $P(\bullet \alpha)=0$, then $P(\alpha \vee \neg \alpha)=1$ and $P(\alpha \wedge$ $\neg \alpha)=0$.

8. $P(\alpha / \beta)+P(\neg \alpha / \beta)=1$, if $P(\circ \alpha)=1$.

9. $P(\beta / \circ \beta)+P(\neg \beta / \circ \beta)=1$.

Proof.

Items 1 to 4 are quite elementary properties coming from the general definition of conditional probability: $P(\alpha / \beta)=\frac{P(\alpha \wedge \beta)}{P(\beta)}$, which gives the alternative product rule $P(\alpha \wedge \beta)=P(\alpha / \beta) \cdot P(\beta)$. The chain rule (item 1 ) is derived by successive applications of product rule. Items 2 to 4 are easy consequences of the definition of conditional probability and clause 5 of Definition 37.

Item 5: since $\alpha \wedge \beta$ is a bottom particle in this case, $P(\alpha \wedge \beta)=0$, and the result follows from 4 .

Item 6 is a consequence of Fact $25(\alpha \wedge \neg \alpha \vdash \bullet \alpha)$, Comparison and elementary inequalities, plus the definition of conditional probability.

Item 7: Easy consequence of Definition 22, R1 (Fact 24) and Comparison (Definition 35).

Item 8: If $P(\circ \alpha)=1$, then by $9 P(\alpha \vee \neg \alpha)=1$, and by Lemma 40 (below) we have $P((\alpha \vee \neg \alpha) \wedge \beta)=P(\beta)=P((\alpha \wedge \beta) \vee(\neg \alpha \wedge \beta))=P(\alpha \wedge \beta)+$ $P(\neg \alpha \wedge \beta)-P(\alpha \wedge \neg \alpha \wedge \beta)$. Since $P(\alpha \wedge \neg \alpha \wedge \beta)=0(P(\circ \alpha)=1$ implies $P(\alpha \wedge \neg \alpha)=0)$, we obtain $P(\beta)=P(\alpha \wedge \beta)+P(\neg \alpha \wedge \beta)$. Dividing both sides by $P(\beta)$ obtains the result, in view of the definition of conditional probability. 
Item 9: In $L E T_{F}, \vdash \circ \beta \leftrightarrow \circ \beta \wedge(\beta \vee \neg \beta) \leftrightarrow(\circ \beta \wedge \beta) \vee(\circ \beta \wedge \neg \beta)$ (proof left to the reader). Thus $P(\circ \beta)=P((\circ \beta \wedge \beta) \vee(\circ \beta \wedge \neg \beta))=P(\circ \beta \wedge \beta)+P(\circ \beta \wedge \neg \beta)$ by Finite Additivity, since $\circ \beta \wedge \beta \wedge \neg \beta$ is a bottom particle (Fact 33). Dividing both sides by $P(\circ \beta)$ yields the result.

\subsection{Independence and incompatibility}

Intuitively, two propositions are independent if the fact that one holds does not have any effect on whether or not the other holds, and vice-versa. Two propositions $\alpha$ and $\beta$, are said to be independent w.r.t. a distribution $P$ if $P(\alpha \wedge \beta)=P(\alpha) \cdot P(\beta)$. Two propositions can be independent relative to one probability distribution and dependent relative to another. Alternatively, independence can be defined as follows: $\alpha$ is independent of $\beta$ if $P(\alpha / \beta)=P(\alpha)$ (or equivalently, $P(\beta / \alpha)=P(\beta)$ ) ${ }^{18}$ Classically, $\alpha$ and $\neg \alpha$ are never independent (unless one of them has probability zero). In view of item 4 of Theorem 42 below, $P(\alpha \wedge \neg \alpha) \leq P(\bullet \alpha)$, hence when $P(\alpha) \cdot P(\neg \alpha)>P(\bullet \alpha), \alpha$ and $\neg \alpha$ are not independent. In this way, $P(\bullet \alpha)$ can be regarded as a bound on the 'degree of independence' between $\alpha$ and $\neg \alpha$.

Intuitively, two propositions $\alpha$ and $\beta$ are logically incompatible if $\alpha$ cannot hold when $\beta$ holds, and vice-versa. Two propositions $\alpha$ and $\beta$, are said to be logically incompatible if $\alpha, \beta \vdash \gamma$, for any $\gamma$, or equivalently, if $\alpha \wedge \beta$ is a bottom particle. Logically incompatible propositions $\alpha$ and $\beta$ with non-zero probabilities are always dependent since $0=P(\alpha \wedge \beta) \neq P(\alpha) \cdot P(\beta)$. Again, for non-zero probabilities, classically $\alpha$ and $\neg \alpha$ are incompatible, and so dependent. In $L E T_{F}$, however, they are neither necessarily incompatible nor necessarily dependent, when $P(\circ \alpha)<1$. We saw in Fact 35 item 1 that $\alpha \wedge \neg \alpha \wedge \circ \alpha$ as well as $\circ \alpha \wedge \bullet \alpha$ defines a bottom particle in $L E T_{F}$. From clause 3 of Definition 37, it follows that for any probability distribution $P, P(\alpha \wedge \neg \alpha \wedge \circ \alpha)=0$ and $P(\circ \alpha \wedge$ $\bullet \alpha)=0$. So, in $L E T_{F} \alpha$ and $\sim \alpha$ are always logically incompatible and hence dependent, while $\alpha$ and $\neg \alpha$ can be independent.

An interesting property concerning the behavior of probability measures in $L E T_{F}$, related to independence in 'extreme cases', occurs when $P(\alpha)=1$. In such cases $\alpha$ is independent from the probability measure of any other distinct proposition $\beta$. This kind of property contributes to the dynamics of evidence, in the sense of the interpretation of preservation of conclusive and non-conclusive evidence in $L E T_{F}$, in such a way that the increasing of conclusive evidence tends to truth.

Lemma 40. Independence of propositions with maximal probability If $P(\alpha)=1$ then $P(\alpha \wedge \beta)=P(\alpha) \cdot P(\beta)$, for $\beta \neq \alpha$

\footnotetext{
${ }^{18}$ Although mathematically equivalent to the former, this characterization of independence by means of conditional probability is debatable, as shown in Fitelson and Hájek (2017), where it is argued that the more general Popperian theory of conditional probability should be adopted, leading to a revision of conventional insights about probabilistic independence. The traditional notions are employed here for mathematical convenience.
} 
Proof. If $P(\alpha)=1$ then $P(\alpha \vee \beta)=1$ from Comparison, since $\alpha \vdash \alpha \vee \beta$. By Finite Additivity $1=P(\alpha \vee \beta)=P(\alpha)+P(\beta)-P(\alpha \wedge \beta)$. As $P(\alpha)=1$, it follows that $P(\alpha \wedge \beta)=P(\beta)$.

The restriction $\alpha \neq \beta$ in the above lemma intends to avoid the problematic cases of 'self-independence' of extreme events. As mentioned before, two events $\alpha$ and $\beta$ are considered to be independent if $P(\alpha \wedge \beta)=P(\alpha) \cdot P(\beta)$, for some probability distribution $P$. This leads to a puzzling situation concerning events $\alpha$ such that $P(\alpha)=0$ or $P(\alpha)=1$. In such cases, $P(\alpha)=P(\alpha \wedge \alpha)=P(\alpha) \cdot P(\alpha)$ in both cases. In this way, extreme probabilities can be regarded as independent of themselves, an uncomfortable situation, as recognized in Fitelson and Hájek (2017).

Lemma 40 leads immediately to the independence of consistent and inconsistent propositions in extreme cases:

1. If $P(\circ \alpha)=1$ then $P(\circ \alpha \wedge \beta)=P(\beta)$, for $\beta \neq \circ \alpha$

2. If $P(\bullet \alpha)=1$ then $P(\bullet \alpha \wedge \beta)=P(\beta)$, for $\beta \neq \bullet \alpha$

3. If $P(\beta)=1$ then $P(\circ \alpha \wedge \beta)=P(\circ \alpha)$, for $\beta \neq \circ \alpha$

4. If $P(\beta)=1$ then $P(\bullet \alpha \wedge \beta)=P(\bullet \alpha)$, for $\beta \neq \bullet \alpha$

Evidence can be increasing or decreasing in an historical series, leading to a dynamic of evidence. This can be expressed in mathematical terms by elementary series. Let $\lim _{i \rightarrow \infty} P_{i}(\alpha)=\lambda$ mean that the sequence of values $P_{1}(\alpha), P_{2}(\alpha), \cdots, P_{i}(\alpha) \cdots$ is strictly monotonous and converges to $\lambda \in[0,1]$.

Lemma 41. The dynamics of evidence

1. If $\lim _{i \rightarrow \infty} P_{i}(\circ \alpha)=1$ or $\lim _{i \rightarrow \infty} P_{i}(\bullet \alpha)=0$, then $\lim _{i \rightarrow \infty} P_{i}(\alpha \vee \neg \alpha)=1$ and $\lim _{i \rightarrow \infty} P_{i}(\alpha \wedge \neg \alpha)=0$.

2. If $\lim _{i \rightarrow \infty} P_{i}(\circ \alpha)=1$ or $\lim _{i \rightarrow \infty} P_{i}(\bullet \alpha)=0$, then $\lim _{i \rightarrow \infty}\left(P_{i}(\alpha)+P_{i}(\neg \alpha)\right)=$ 1.

Proof. Suppose $\lim _{i \rightarrow \infty} P_{i}(\circ \alpha)=1$; by $P E M^{\circ}$ and Comparison, $P_{i}(\circ \alpha) \leq P_{i}(\alpha \vee$ $\neg \alpha) \leq 1$. By the Squeeze Theorem of elementary calculus for series (aka the Sandwich Theorem) $\lim _{i \rightarrow \infty} P_{i}(\alpha \vee \neg \alpha)=1$. All other limits are proved in similar ways.

The meaning of Lemma 41 is precisely that the values of $P_{i}(\circ \alpha)$ can be interpreted as degrees of classicality, in the sense that greater values of $P_{i}(\circ \alpha)$ indicate that the situation is approaching classicality and, conversely, the values of $P_{i}(\bullet \alpha)$ can be interpreted as degrees of anticlassicality, in the sense that smaller values of $P_{i}(\bullet \alpha)$ indicate that the situation is approaching classicality.

Some useful (though almost all immediate) properties of $L E T_{F}$-distributions are the following: 


\section{Theorem 42.}
1. If $\alpha \dashv \beta$, then $P(\alpha)=P(\beta)$.

2. $P(\alpha \vee \beta)=P(\alpha)+P(\beta)$, if $\alpha$ and $\beta$ are logically incompatible.

3. $P(\alpha \vee \beta \vee \gamma)=P(\alpha)+P(\beta)+P(\gamma)-P(\alpha \wedge \beta)-P(\alpha \wedge \gamma)-P(\beta \wedge \gamma)+P(\alpha \wedge \beta \wedge \gamma)$.

4. $P(\alpha \wedge \neg \alpha) \leq P(\bullet \alpha)$.

5. $P(\circ \alpha) \leq P(\alpha \vee \neg \alpha)$.

6. $P(\circ \alpha)=1-P(\bullet \alpha)$.

7. $P(\oplus \alpha \wedge \neg \alpha)=0, P(\sim \alpha \wedge \alpha)=0$.

8. $P(\circ \alpha \vee \bullet \alpha)=1, P(\alpha \vee \neg \alpha \vee \bullet \alpha)=1$

9. $P(\circ \alpha \vee(\alpha \wedge \neg \alpha)) \leq P(\alpha \vee \neg \alpha)$

10. $1+P((\alpha \vee \neg \alpha) \wedge \bullet \alpha)=P(\alpha \vee \neg \alpha)+P(\bullet \alpha)$

11. $1+P((\alpha \wedge \neg \alpha) \vee \circ \alpha)=P(\alpha \wedge \neg \alpha)+P(\circ \alpha)$

12. If $P(\circ \alpha)=1$ (or equivalently $P(\bullet \alpha)=0$ ), then $P(\neg \alpha)=1-P(\alpha)$

13. If $P(\circ \alpha)=1$ (or equivalently $P(\bullet \alpha)=0)$, then $P(\alpha \vee \neg \alpha)=1$ and $P(\alpha \wedge$ $\neg \alpha)=0$.

Proof. Routine, from the axioms of probability and the derivability relation of $L E T_{F}$. We just sketch the proof of items 12 and 13. For 12, suppose $P(\circ \alpha)=1$; by items 4 and 5 above, $P E M^{\circ}$, and Comparison, $1=P(\circ \alpha) \leq P(\alpha \vee \neg \alpha)$, and $P(\alpha \wedge \neg \alpha) \leq P(\bullet \alpha)=0$, hence by Finite Additivity $P(\alpha \vee \neg \alpha)+P(\alpha \wedge \neg \alpha)=$ $1+0=P(\alpha)+P(\neg \alpha)$. Hence $P(\neg \alpha)=1-P(\alpha)$. For 13 a similar reasoning as of 12 is obtained.

Items 1 and 2 are usual results in probabilistic logic, and 3 is a particular case of the Inclusion-Exclusion property for finite probability, easily adapted for propositions, that hold for arbitrary finite disjunctions (see Grinstead and Snell (1997)). Items 4 and 5 establish constraints on the values of $P(\circ \alpha)$, $P(\alpha)$ and $P(\neg \alpha)$. Item 7 concerns bottom particles, and 8, theorems of $L E T_{F}$ essential for proving total probability theorems (Section 4.3 below). Items 12 and 13 show the classical behavior of probabilities when $P(\circ \alpha)=1$. 


\subsection{Total probability theorems for $L E T_{F}$}

In the classical approach to probability, total probability theorems compute the probability of an event $\beta$ in a sample space partitioned into exclusive and exhaustive events. Typically, for a partition in two pieces, a total probability theorem that reflects excluded middle assumes the following form:

$$
P(\beta)=P(\beta \wedge \alpha)+P(\beta \wedge \neg \alpha) .
$$

Here, however, we are not really talking about sample spaces, about events themselves, but rather about the information related to such events, that we call an information space. In the standard approach to probability theory, we start from a group of events, say, the two outcomes of tossing a coin, and attribute probabilities to these events, whose sum is always equal to 1 . Let $\alpha$ express that the toss of a coin comes up heads. The sample space is thus divided into two parts, $\alpha$ and $\neg \alpha$, corresponding respectively to heads and tails (not heads). If the coin behaves as expected, their probability are the same.

On the other hand, we consider here a language that is able to express information about some event - for example, the result of a referendum - that comes from different sources and may be unreliable. Such information is constituted by evidence for 'yes' and for 'no' that can be non-conclusive, incomplete, contradictory, more reliable or less reliable, and perhaps even conclusive. Let $\alpha$ express the result 'yes', and $\neg \alpha$ the result 'no'. In this case, the propositions we are concerned with are $\alpha, \neg \alpha, \circ \alpha, \bullet \alpha$, as well as other propositions of the language of $L E T_{F}$ formed from them, for example, $\bullet \alpha \vee \alpha, \alpha \wedge \neg \alpha, \circ \alpha \wedge \alpha$, etc. A $L E T_{F}$-probability distribution attributes values to these propositions. The information space is thus constituted by such propositions and the measures of probabilities attributed to them by a $L E T_{F}$-probability distribution $P$. Note that, contrary to the classical case, $P(\alpha)+P(\neg \alpha)$ can be greater or less than 1 precisely because $\alpha$ and $\neg \alpha$ do not establish a partition of the information space.

Now, the question is: since we cannot rely on the classical, mutually exclusive partitions of the sample space, how can total probability theorems be stated? In order to provide such theorems for $L E T_{F}$, we have to rely on the connectives $\circ$, -, and on the connectives defined in Fact 34. We also need a bit of terminology.

Definition 43. (Cleavage)

Let us call a cleavage a (finite) family of propositions $\left\{\alpha_{1}, \alpha_{2}, \ldots, \alpha_{n}\right\}$. A cleavage is said to be exhaustive if $\alpha_{1} \vee \alpha_{2} \vee \ldots \vee \alpha_{n}$ is a tautology, and so it covers all the information space, possibly with intersections. A cleavage is said to be exclusive when $\alpha_{1} \vee \alpha_{2} \vee \ldots \vee \alpha_{n}$ are pairwise logically incompatible. In this case, it does not yield intersection of information (in the sense that $\alpha_{i} \wedge \alpha_{j}$ for $i \neq j$ is a bottom particle), and possibly does not cover the whole space. An exhaustive and exclusive cleavage is a partition.

Items 2 to 5 of Theorem 36 cleave the information space exhaustively but not exclusively. Items 1 and 6 , on the other hand, cleave the information space in parts that are exhaustive and exclusive, and so they are partitions. Notice 
that item 3 of Theorem 36 corresponds to the six scenarios of conclusive and non-conclusive evidence that we have seen in Section 3.1. These scenarios can be graphically represented as follows:

\begin{tabular}{|c|c|c|c|c|c|}
\hline$\bullet \alpha \wedge \alpha$ & $\bullet \alpha \wedge \neg \alpha$ & $\bullet \alpha$ & $\bullet \alpha \wedge(\alpha \wedge \neg \alpha)$ & $\circ \alpha \wedge \alpha$ & $\circ \alpha \wedge \neg \alpha$ \\
\hline 1 & 2 & 3 & 4 & 5 & 6 \\
\hline
\end{tabular}

Item 1 of Theorem 36 above emphasizes the division between non-conclusive evidence (scenarios 1 to 4 ) and conclusive evidence (scenarios 5 and 6), while item 6 , in addition, splits the conclusive evidence into truth (5) and falsity (6). These propositions can be understood as expressing different ways we can look at the information space. The following total probability theorems can be obtained depending upon certain cleavages, based on Theorem 36 .

Theorem 44. Total probability theorems

1. $P(\beta)=P(\beta \wedge \circ \alpha)+P(\beta \wedge \bullet \alpha)$, w.r.t. the cleavage $\{\circ \alpha, \bullet \alpha\}$.

2. $P(\beta)=P(\beta \wedge \alpha)+P(\beta \wedge \neg \alpha)+P(\beta \wedge \bullet \alpha)-P(\beta \wedge \alpha \wedge \bullet \alpha)-P(\beta \wedge \neg \alpha \wedge \bullet \alpha)$, w.r.t. the cleavage $\{\alpha, \neg \alpha, \bullet \alpha\}$.

3. $P(\beta)=P(\beta \wedge \circ \alpha \wedge \alpha)+P(\beta \wedge \circ \alpha \wedge \neg \alpha)+P(\beta \wedge \bullet \alpha)-P(\beta \wedge \bullet \alpha \wedge \alpha \wedge \neg \alpha)$, w.r.t. cleavage $\{\bullet \alpha \wedge \alpha, \bullet \alpha \wedge \neg \alpha, \bullet \alpha, \bullet \alpha \wedge \alpha \wedge \neg \alpha, \circ \alpha \wedge \alpha, \circ \alpha \wedge \neg \alpha\}$.

4. $P(\beta)=P(\beta \wedge \alpha)+P(\beta \wedge \neg \alpha)+P(\beta \wedge \ominus \alpha)-P(\beta \wedge \alpha \wedge \ominus \alpha)-P(\beta \wedge \neg \alpha \wedge \ominus \alpha)$, w.r.t. the cleavage $\{\alpha, \neg \alpha, \ominus \alpha\}$.

5. $P(\beta)=P(\beta \wedge \alpha)+P(\beta \wedge \neg \alpha)+P(\beta \wedge \approx \alpha)-P(\beta \wedge \alpha \wedge \approx \alpha)-P(\beta \wedge \neg \alpha \wedge \approx \alpha)$, w.r.t. the cleavage $\{\alpha, \neg \alpha, \approx \alpha\}$.

6. $P(\beta)=P(\beta \wedge \oplus \alpha)+P(\beta \wedge \sim \alpha)+P(\beta \wedge \bullet \alpha)$, w.r.t. the cleavage $\{\oplus \alpha, \sim \alpha, \bullet \alpha\}$.

Proof. 1. $\beta \dashv \vdash(\beta \wedge \circ \alpha) \vee(\beta \wedge \bullet \alpha)$. So, $P(\beta)=P((\beta \wedge \circ \alpha) \vee(\beta \wedge \bullet \alpha))=$ $P(\beta \wedge \circ \alpha)+P(\beta \wedge \bullet \alpha)-P(\beta \wedge \circ \alpha \wedge \bullet \alpha)=P(\beta \wedge \circ \alpha)+P(\beta \wedge \bullet \alpha)$. The remaining proofs are left to the reader. In view of Definition 3.1 (connectives $\oplus, \sim, \ominus, \approx)$, some of these cleavages are equivalent.

\subsection{Bayes' rule}

As is well-known, Bayes' rule, or Bayes' theorem, computes the probability of an event based on previous information related to that event. The standard Bayes' rule proves that, for $P(\beta) \neq 0$ :

$$
P(\alpha / \beta)=\frac{P(\beta / \alpha) \cdot P(\alpha)}{P(\beta)}
$$

In the equation above, interpreted in terms of measures of evidence rather than standard probabilities, $P(\alpha)$ denotes the evidence available for $\alpha$ without taking 
into consideration any evidence for $\beta$. The latter is supposed to affect someway the evidence for $\alpha$, and so $P(\alpha / \beta)$ is the measure of the evidence for $\alpha$ after $\beta$ is taken into account. $P(\beta / \alpha)$, usually called the 'likelihood' in probability theory, is the evidence for $\beta$ when $\alpha$ is considered as given, and $P(\beta)$, usually called the 'marginal likelihood', is the total evidence available for $\beta$, that takes into account all the possible cases where $\beta$ may occur. In what follows, we define some relevant versions of Bayes' rule. Differently from the classical case, these versions are not equivalent. They show how the notion of classicality can modify Bayesian probability updating.

Theorem 45. Bayes' Conditionalization Rules

1.

$$
\begin{gathered}
P(\alpha / \beta)=\frac{P(\beta / \alpha) \cdot P(\alpha)}{P(\beta / \circ \alpha) \cdot P(\circ \alpha)+P(\beta / \bullet \alpha) \cdot P(\bullet \alpha)} \\
\quad \text { for } P(\beta) \neq 0, P(\circ \alpha) \neq 0, \text { and } P(\bullet \alpha) \neq 0 .
\end{gathered}
$$

Proof. From the definition of conditional probability and Theorem 44 item 1.

2.

$$
\begin{aligned}
P(\alpha / \beta)= & \frac{P(\beta / \alpha) \cdot P(\alpha)}{P(\beta / \alpha) \cdot P(\alpha)+P(\beta / \neg \alpha) \cdot P(\neg \alpha)+P(\beta / \bullet \alpha) \cdot P(\bullet \alpha)-} \\
& P(\beta / \alpha \wedge \bullet \alpha) \cdot P(\alpha \wedge \bullet \alpha)-P(\beta / \neg \alpha \wedge \bullet \alpha) \cdot P(\neg \alpha \wedge \bullet \alpha)
\end{aligned}
$$

Proof. From the definition of conditional probability and Theorem 44 . item 2.

3.

$$
\begin{gathered}
P(\alpha / \beta)=\frac{P(\beta / \alpha) \cdot P(\alpha)}{P(\beta / \oplus \alpha) \cdot P(\oplus \alpha)+P(\beta / \sim \alpha) \cdot P(\sim \alpha)+P(\beta / \bullet \alpha) \cdot P(\bullet \alpha)} \\
\quad \text { for } P(\beta) \neq 0, P(\bullet \alpha) \neq 0, P(\oplus \alpha) \neq 0, \text { and } P(\ominus \alpha) \neq 0 .
\end{gathered}
$$

Proof. From the definition of conditional probability and Theorem 44 . item 6.

It should be clear that the process of limit can be easily established for the above formulations of Bayes' rules. If $\lim P_{i}(\circ \alpha)=1$ (or equivalently $\lim P_{i}(\bullet \alpha)=0$ ) then item 1 above reduces to $P(\circ \alpha / \beta)=1$. Analogously, if $\lim P_{i}(\bullet \alpha)=0$ (or equivalently $\left.\lim P_{i}(\circ \alpha)=1\right)$, then items 2 and 3 above reduce to the standard form of Bayes' rule. 


\section{$5 \quad$ Final remarks}

This paper has been conceived to be a further development of the approach to paraconsistency as preservation of evidence presented in Carnielli and Rodrigues (2017, 2019), where an interpretation of contradictions in terms of nonconclusive evidence was proposed. The underlying assumption is that there are no true contradictions, but rather argumentative contexts in which conflicting evidence, as well as the absence of any evidence, may occur. The valuation semantics is able to express only that there is or there is not evidence for a proposition $\alpha$, while the probabilistic semantics presented here intends to express the degree of evidence enjoyed by a given proposition. The acceptance of scenarios in which $P(\alpha)+P(\neg \alpha)>1$, however, does not mean that there may be something like 'contradictory sample spaces', or 'contradictory probabilistic spaces'. The latter would be the probabilistic counterpart of contradictions in reality, a view on paraconsistency not endorsed by us. In our view, it is the information available about some collection of events that can be contradictory. So, instead of talking about sample spaces, the concept of an information space has been introduced here.

Both $L E T_{J}$ and $L E T_{F}$ are Logics of Formal Inconsistency and Undeterminedness suitable for an intuitive interpretation in terms of preservation of evidence and truth. The intuition regarding $\circ$ and $\bullet$ as "classically contradictory' w.r.t. each other had already been presented in Carnielli, Coniglio, and Rodrigues (2019, Section 4.4). $L E T_{F}$, however, as far as we know, is the first formal system where these connectives are both primitive and have the deductive behavior given by rules Cons and Comp, that are in some sense analogous to explosion and excluded middle. The connective $\bullet$, and the fact that $\circ \alpha \vee \bullet \alpha$ and $\alpha \vee \neg \alpha \vee \bullet \alpha$ are theorems of $L E T_{F}$, are essential for proving total probability theorems and Bayes' rules (Theorems 44 and 45 .

The probabilistic semantics of $L E T_{F}$ has been axiomatically stated in definitions 37 and 38 . Accordingly, $P(\alpha)+P(\neg \alpha)$ can be greater or less than 1 , and this is interpreted as scenarios, respectively, of conflicting evidence, and little or no evidence. When $P(\circ \alpha)=1$, the classical behavior of $P(\alpha)$ and $P(\neg \alpha)$ is restored, and this is interpreted as saying that the evidence available for $\alpha$ and $\neg \alpha$ is subjected to the laws of standard probability theory. But $P(\circ \alpha)$ may be less than 1, and in this case, according to the axioms, it expresses the degree to which $P(\alpha)$ and $P(\neg \alpha)$ are expected to behave classically (the value of $P(\circ \alpha)$ establishes constraints on the values of $P(\alpha \vee \neg \alpha)$ and $P(\alpha \wedge \neg \alpha)$, cf. Lemma 41). Accordingly, $P(\circ \alpha)<1$ can be intuitively interpreted as expressing the reliability of the available evidence for $\alpha$ and $\neg \alpha$ : greater reliability corresponds to a greater degree of classicality.

Our treatment here does not intend to express degrees of belief by means of probability measures. The notion of evidence for $\alpha$, as explained in Section 2.2.1 does not imply belief in $\alpha$. So, the degree of evidence for $\alpha$ measured by a statement $P(\alpha)=\epsilon$ is not a measure of the belief of an agent in $\alpha$. However, nothing a fortiori prevents the formal system proposed here, together with its probabilistic semantics, of being interpreted, or used, as a tool to measure 
degrees of belief, uncertainty, or some other relation between agents and propositions. Similar remarks apply to the connective $\circ$. In $P(\circ \alpha)=\epsilon$, the value of $\epsilon$ expresses the degree to which it is expected that $P(\alpha)$ behaves classically. Indeed, $\epsilon$ can also be interpreted as the degree of reliability of evidence for $\alpha$, coherence with previous data or with a historical series of measures of evidence for $\alpha$, or even with a subjective ingredient, for example, as the degree of trustfulness of the belief in $\alpha$, or certainty/uncertainty of $\alpha$.

The rules for $\circ$ and $\bullet$, due to their dual character, show a symmetry that deserves to be further investigated from the proof-theoretic point of view. There are some extensions of $L E T_{J}$ and $L E T_{F}$ that also deserve to be studied. The operator • and the rules Cons and Comp can be added to $L E T_{J}$, obtaining a logic that differs from $L E T_{F}$ only in the implication for the non-classical propositions. Two intuitively appealing equivalences are the following:
1. $\circ \alpha \dashv \vdash \circ \circ \alpha$
2. $\circ \alpha \dashv \vdash \circ \neg \alpha$

It was shown in Carnielli and Rodrigues (2017, Fact 17) that $L E T_{J}$ has no theorems of the form $\circ \alpha$ (the same result also holds for $L E T_{F}$ ), and it was argued that $L E T_{J}$ (and so $L E T_{F}$ ) was conceived in such a way that $\circ$ has to be introduced from outside the formal system. This is in line with the idea that information about conclusive evidence for a proposition $\alpha$ comes from outside the formal system. But it is also very reasonable to suppose that once the truth value of a proposition $\alpha$ has been established, and so $\circ \alpha$ holds and $\alpha$ has classical behavior, then $\circ \alpha, \circ \circ \alpha$, and so on, also have classical behavior. Conversely, it is also reasonable to conclude $\circ \alpha$ from $\circ \circ \alpha$, and so on. These ideas are expressed by 1 above. The equivalence 2 above makes explicit inside the system the first part of the result achieved by Fact 31 (to wit: once $\circ \neg^{n} \alpha$ is proved, and so it follows that $\neg^{n} \alpha$ is subjected to classical logic, for any formula $\neg^{m} \alpha, m \geq 0$, $\neg^{m} \alpha$ is also subjected to classical logic). Valuation semantics for these rules are straightforward, and adding these rules would produce a decidable formal system.

We believe that the probabilistic semantic relation presented in Section 4 will succeed as a tool for dealing with real argumentative contexts, including investigative scenarios and databases concerned with different degrees of evidence attributed to propositions. But this claim needs to be further investigated.

\section{References}

P. Achinstein. Concepts of evidence. In Evidence, Explanation, and Realism. Oxford University Press, 2010a.

P. Achinstein. Why philosophical theories of evidence are (and ought to be) ignored by scientists. In Evidence, Explanation, and Realism. Oxford University Press, 2010b. 
A. R. Anderson and N. Belnap. Entailment: The Logic of Relevance and Necessity (Vol. 1). Princeton University Press, 1975.

A. R. Anderson and N. D. Belnap. First degree entailments. Mathematische Annalen, 149:302-319, 1963.

A. R. Anderson, N. Belnap, and Dunn J. M. Entailment: The Logic of Relevance and Necessity (Vol. 2). Princeton University Press, 1992.

N. D. Belnap. How a computer should think. In Contemporary Aspects of Philosophy (ed. G. Ryle). Oriel Press, 1977a.

N. D. Belnap. A useful four-valued logic. In Modern Uses of Multiple Valued Logics (eds. Epstein, G. and Dunn, J. M.). D. Reidel, Dordrecht, 1977b.

J. Bueno-Soler and W. Carnielli. Paraconsistent probabilities: Consistency, contradictions and Bayes' theorem. In J. Stern, editor, Special Issue Statistical Significance and the Logic of Hypothesis Testing. Entropy 18(9), 2016. Open access http://www.mdpi.com/1099-4300/18/9/325/htm

J. Bueno-Soler and W. Carnielli. Paraconsistent probabilities, their significance and their uses. In P. Gouveia, C. Caleiro, and F. Donisio, editors, Logic and Computation: Essays in Honour of Amilcar Sernadas, pages 197-230. College Publications, 2017.

C. Caleiro, W. Carnielli, M. E. Coniglio, and J. Marcos. Two's company: "The Humbug of Many Logical Values". Logica Universalis, 2:175-194, 2005.

W. Carnielli and A. Rodrigues. An epistemic approach to paraconsistency: a logic of evidence and truth. Synthese, 196:3789-3813, 2017. doi: 10.1007/ s11229-017-1621-7.

W. Carnielli and A. Rodrigues. On epistemic and ontological interpretations of intuitionistic and paraconsistent paradigms. Logic Journal of the IGPL, 2019. doi: $10.1093 /$ jigpal/jzz041.

W. Carnielli, M. E. Coniglio, and J. Marcos. Logics of formal inconsistency. In Gabbay and Guenthner, editors, Handbook of Philosophical Logic, volume 14. Springer, 2007.

W. Carnielli, M. Coniglio, and A. Rodrigues. Recovery operators, paraconsistency and duality. Logic Journal of the IGPL, 2019. doi: 10.1093/jigpal/ jzy054.

N. C. A. da Costa and E. Alves. A semantical analysis of the calculi $\mathrm{C}_{n}$. Notre Dame Journal of Formal Logic, 18(4):621-630, 1977.

L. Demey, B. Kooi, and J. Sack. Logic and probability. Stanford Encyclopedia of Philosophy, 2013. URL https://plato.stanford.edu/archives/sum2017/ entries/logic-probability/. 
J. M. Dunn. Intuitive semantics for first-degree entailments and 'coupled trees'. Philosophical Studies, 29(3):149-168, 1976.

J. M. Dunn. Information in computer science. In P. Adriaans \& J. van Benthem, editor, Philosophy of Information. Volume 8 of Handbook of the Philosophy of Science, pages 581-608. Elsevier, 2008.

B. Fitelson and A. Hájek. Declarations of independence. Synthese, 194(10): 3979-3995, 2017.

M. Fitting. Paraconsistent logic, evidence, and justification. Studia Logica, 105 (6):1149-1166, 2016a.

M. Fitting. Justifications that might be wrong (abstract). In Modalities, Conditionals, and Values: A Symposium on Philosophical Logic in Celebration of the Centenary of Georg Henrik von Wright. May 23-25, 2016, Helsinki, Fin-

land, 2016b. URL https://www.cs.helsinki.fi/u/hakli/vw/abstracts. html.

C. M. Grinstead and J. L. Snell. Introduction to Probability. American Mathematical Society, Providence, RI, 1997.

T. Kelly. Evidence. The Stanford Encyclopedia of Philosophy (Fall 2014, ed. E. Zalta), 2014.

O. Lahav, J. Marcos, and Y. Zohar. It ain't necessarily so: basic sequent systems for negative modalities. In L. Beklemishev, S. Demri, and A. Máté, editors, Advances in Modal Logic. College Publications, London, 2016.

A. Loparic. A semantical study of some propositional calculi. The Journal of Non-Classical Logic, 3(1):73-95, 1986.

A. Loparic. Valuation semantics for intuitionistic propositional calculus and some of its subcalculi. Principia, 14(1):125-133, 2010.

A. Loparic and E. Alves. The semantics of the systems $\mathrm{C}_{n}$ of da Costa. In A. I. Arruda, N. C. A. da Costa, and A. M. Sette, editors, Proceedings of the III Brazilian Conference on Mathematical Logic, Recife, 1979, pages 161-172. Brazilian Logic Society, São Paulo, 1979.

J. Łukasiewicz. Die logischen Grundlagen der Wahrscheinlichkeitsrechnung. Spólka Wydawnicza Polska, Krakow, 1913.

J. Marcos. Nearly every normal modal logic is paranormal. Logique et Analyse, 48:279-300, 2005.

H. Omori and H. Wansing. 40 Years of FDE: An Introductory Overview. Studia Logica, Special Issue "40 Years of FDE", 105:1021-1049, 2017. doi: 10.1007/ s11225-017-9748-6. 
G. Shafer. A Mathematical Theory of Evidence. Princeton University Press, 1976.

A. Tarski. Wahrscheinlichkeitslehre und mehrwertige Logik. Erkenntnis, 5: $174-175,1935$.

D. van Dalen. Logic and Structure. Springer, 4th edition, 2008.

B. Weatherson. From classical to intuitionistic probability. Notre Dame Journal of Formal Logic, 44:111-123, 2003.

L. Zadeh. Review of Glenn Shafer, A Mathematical Theory of Evidence. AI Magazine, 5(3):81-83, 1984. 